\title{
WEIGHTED PUSHDOWN SYSTEMS WITH INDEXED WEIGHT DOMAINS *
}

\author{
YASUHIKO MINAMIDE \\ Department of Mathematical and Computing Sciences, Tokyo Institute of Technology, Japan \\ e-mail address: minamide@is.titech.ac.jp
}

\begin{abstract}
The reachability analysis of weighted pushdown systems is a very powerful technique in verification and analysis of recursive programs. Each transition rule of a weighted pushdown system is associated with an element of a bounded semiring representing the weight of the rule. However, we have realized that the restriction of the boundedness is too strict and the formulation of weighted pushdown systems is not general enough for some applications.

To generalize weighted pushdown systems, we first introduce the notion of stack signatures that summarize the effect of a computation of a pushdown system and formulate pushdown systems as automata over the monoid of stack signatures. We then generalize weighted pushdown systems by introducing semirings indexed by the monoid and weaken the boundedness to local boundedness.
\end{abstract}

\section{INTRODUCTION}

The reachability analysis of weighted pushdown systems is a very powerful technique in verification and analysis of recursive programs [RSJM05]. Each transition rule of a weighted pushdown system is associated with an element of a semiring representing the weight of the rule. To guarantee termination of the analysis, the semiring of the weight must be bounded: there should be no infinite descending sequence of weights. However, recently, we have realized that this restriction of the boundedness is too strict and the formulation of weighted pushdown systems is not general enough for some applications. For the two applications below, the standard algorithm for the reachability analysis of weighted pushdown systems actually works and terminates. However, they require semirings that are not bounded and thus the standard framework of weighted pushdown systems cannot guarantee termination.

The first application is the reachability analysis of conditional pushdown systems. Conditional pushdown systems extend pushdown systems with the ability to check the whole stack content against a regular language [EKS03, LO10]. We proposed an algorithm of their reachability analysis in our previous work on the analysis of the HTML 5 parser

2012 ACM CCS: [Theory of computation]: Formal languages and automata theory; Semantics and reasoning-Program reasoning-Program analysis.

Key words and phrases: pushdown system, reachability analysis, semiring.

* A preliminary version of this article appeared in the proceedings of the 19th International Conference on Tools and Algorithms for the Construction and Analysis of Systems (TACAS), LNCS 7795, pp. 230-244, 2013. 
specification MM12. After the development of the algorithm, we realized that the algorithm can be considered as the reachability analysis of weighted pushdown systems. However, it required an unbounded semiring.

The second application is the analysis of recursive programs with local variables. For the efficient analysis of recursive programs, Suwimonteerabuth proposed an encoding of local variables into weight implemented with BDDs [Suw09]. The weight has a structure depending on a configuration of stack and requires a semiring that is not bounded.

To generalize weighted pushdown systems, we first introduce stack signatures that summarize the effect of a computation of a pushdown system as a pair of words over a stack alphabet. A stack signature $w_{1} / w_{2}$ represents a computation of a pushdown system that pops $w_{1}$ and pushes $w_{2}$ as its total effect. We show that the set of stack signatures forms an ordered monoid, i.e., a monoid that is equipped with a partial order compatible with the multiplication of the monoid. We then formulate pushdown systems as automata over the monoid of stack signatures.

We extend the structure of weight by introducing semirings indexed by a monoid element. An indexed semiring $\mathcal{S}$ over a monoid $\mathcal{M}$ has domains $D_{m}$ indexed by $m \in \mathcal{M}$ and indexed operations $\otimes_{m, m^{\prime}}: D_{m} \times D_{m^{\prime}} \rightarrow D_{m m^{\prime}}$ and $\oplus_{m}: D_{m} \times D_{m} \rightarrow D_{m}$ for $m, m^{\prime} \in \mathcal{M}$. The operations must satisfy the properties of semirings extended to indexed domains. Weighted pushdown systems are then generalized to those over a semiring indexed by the monoid of stack signatures. We show that the reachability analysis of weighted pushdown systems by Reps et al. [RSJM05] can be refined to those over an indexed semiring and the boundedness can be replaced with the local boundedness.

To prove that a structure forms an indexed semiring, we need to show many properties on its multiplication and addition. It is rather cumbersome to prove them from scratch. We show that an indexed semiring can be constructed from a simplified structure, called a weight structure. All the indexed semirings used in our applications of weighted pushdown systems are presented as weight structures. It is much easier to show a structure forms a weight structure.

We present several applications of pushdown systems with indexed weighted domains. The first application is an encoding of a pushdown system into a weighted pushdown system whose stack alphabet is a singleton. This is a simplified version of the encoding of local variables into weight by Suwimonteerabuth [Suw09]. The second application is an indexed semiring to encode the reachability analysis of conditional pushdown systems into that of weighted pushdown systems. We also consider the coverability in well-structured pushdown systems by Cai and Ogawa [CO13, and the reachability in pushdown systems with stack manipulation by Uezato and Minamide [UM13. Since the indexed semirings used in these applications are locally bounded, our framework guarantees termination of the analyses.

This paper is organized as follows. Section 2 reviews the definitions of semirings and weighted automata. In Section 3 , we introduce stack signatures that summarize the effect of a computation of a pushdown system and show that they form a semiring. In Section 4, we introduce semirings indexed by a monoid and weighted automata are extended to those over an indexed semiring. Section 5 introduces weighted pushdown automata over an indexed semiring and extends the standard saturation procedure to them. Section 6 presents a simplified structure to easily construct a semiring indexed by a monoid. Several applications of our framework are presented in Section 7. Finally, we discuss related work and conclude. 


\section{Semirings and Weighted Automata}

We first review the definitions of semirings and weighted automata.

Definition 2.1. A semiring is a structure $\mathcal{S}=\langle D, \oplus, \otimes, 0,1\rangle$ where $D$ is a set, 0 and 1 are elements of $D, \oplus$ and $\otimes$ are binary operations on $D$ such that

(1) $\langle D, \oplus, 0\rangle$ is a commutative monoid.

(2) $\langle D, \otimes, 1\rangle$ is a monoid.

(3) $\otimes$ distributes over $\oplus$.

$$
(x \oplus y) \otimes z=(x \otimes z) \oplus(y \otimes z) \quad x \otimes(y \oplus z)=(x \otimes y) \oplus(x \otimes z)
$$

(4) 0 is an annihilator with respect to $\otimes: 0 \otimes x=0=x \otimes 0$ for all $x \in D$.

We say that a semiring $\mathcal{S}$ is idempotent if its addition $\oplus$ is idempotent (i.e., $a \oplus a=a$ ). For an idempotent semiring $\langle D, \oplus, \otimes, 0,1\rangle,\langle D, \oplus\rangle$ can be considered as a join semilattic $\left.\right|^{1}$. Then, the partial order $\sqsubseteq$ is defined by $a \sqsubseteq b$ iff $a \oplus b=b$ for an idempotent semiring. We say that an idempotent semiring is bounded if there are no infinite ascending chains with respect to $\sqsubseteq$.

In this paper, we consider weighted automata without initial and final states.

Definition 2.2. A weighted automaton $\mathcal{A}$ over an idempotent semiring $\mathcal{S}$ and an alphabet $\Gamma$ is a structure $\langle\Gamma, Q, E\rangle$ where $Q$ is a finite set of states, $E: Q \times \Gamma \times Q \rightarrow \mathcal{S}$ is a set of transition rules each of which associates an element in $\mathcal{S}$ as weight.

For weighted automata over an alphabet $\Gamma$ and a semiring $\mathcal{S}=\langle D, \oplus, \otimes, 0,1\rangle$, we introduce the transition relation of the form $q \stackrel{w \mid a}{\longrightarrow} q^{\prime}$ where $w \in \Gamma^{*}$ and $a \in D$. It is inductively defined as follows.

- $q \stackrel{\epsilon \mid 1}{\longrightarrow} q$ for any $q \in Q$.

- $q \stackrel{\gamma \mid a}{\longrightarrow} q^{\prime}$ if $a=E\left(\left\langle q, \gamma, q^{\prime}\right\rangle\right)$.

- $q \stackrel{w w^{\prime} \mid a \otimes b}{\longrightarrow} q^{\prime}$ if $q \stackrel{w \mid a}{\longrightarrow} q^{\prime \prime}$ and $q^{\prime \prime} \stackrel{w^{\prime} \mid b}{\longrightarrow} q^{\prime}$.

Then, for two states $q$ and $q^{\prime}$ and a word $w$, we consider the total weight of the transitions of the form $q \stackrel{w \mid a}{\longrightarrow} q^{\prime}$ defined as follows 2 .

$$
\delta\left(q, w, q^{\prime}\right)=\bigoplus\left\{a \mid q \stackrel{w \mid a}{\longrightarrow} q^{\prime}\right\}
$$

This is well-defined because there are only finitely many transitions of this form and we assume that the semiring is idempotent. In the general theory of weighted automata, we do not impose that the semiring is idempotent [ÉK09]. However, we impose the condition to adopt the simple and intuitive definition above.

\section{Stack Signatures}

We introduce stack signatures that summarize the effect of a transition on stack as a pair of words over a stack alphabet. It is shown that the set of stack signatures forms a monoid, and then a semiring by introducing a partial order on them. Stack signatures naturally appear

\footnotetext{
${ }^{1}$ In RSJM05, it is considered as a meet semilattice.

${ }^{2}$ This is basically a formal power series, which is used to define the behaviour of weighted automata [ÉK09].
} 
in the theory of context-free grammars and pushdown systems [Suw09, MT06, TM07]. We adopt the term 'stack signature' introduced by Suwimonteerabuth [Suw09].

The proofs of most results in this section appear in Appendix A. They are not fundamentally difficult, but require detailed case-analysis. Thus, we also formalized stack signatures and proved their properties in Isabelle/HOL by extending our previous work on a formalization of decision procedures on context-free grammars Min07 $]^{3}$

The effect of a transition of a pushdown system can be summarized as a pair of sequences of stack symbols written $w_{1} / w_{2}$ where $w_{1}$ are the symbols popped by the transition and $w_{2}$ are those pushed by the transition. We consider that pushing $\gamma$ and then popping the same $\gamma$ cancel the effect, but popping $\gamma$ and then pushing $\gamma$ have the effect $\gamma / \gamma$.

Definition 3.1. We call elements of $\Gamma^{*} \times \Gamma^{*}$ stack signatures and write $w / w^{\prime}$ for a stack signature $\left\langle w, w^{\prime}\right\rangle$.

- We say that $w_{1} / w_{1}^{\prime}$ and $w_{2} / w_{2}^{\prime}$ are compatible if either $w_{1}^{\prime}$ is a prefix of $w_{2}$ or $w_{2}$ is a prefix of $w_{1}^{\prime}$. Furthermore, they are called strictly compatible if $w_{1}^{\prime}=w_{2}$.

- For compatible $w_{1} / w_{1}^{\prime}$ and $w_{2} / w_{2}^{\prime}$, we define $w_{1} / w_{1}^{\prime} \cdot w_{2} / w_{2}^{\prime}$ by

$$
w_{1} / w_{1}^{\prime} \cdot w_{2} / w_{2}^{\prime}= \begin{cases}w_{1} / w_{2}^{\prime} w_{1}^{\prime \prime} & \text { if } w_{1}^{\prime}=w_{2} w_{1}^{\prime \prime} \\ w_{1} w_{2}^{\prime \prime} / w_{2}^{\prime} & \text { if } w_{2}=w_{1}^{\prime} w_{2}^{\prime \prime}\end{cases}
$$

For example, we have $\gamma_{1} / \gamma_{2} \cdot \gamma_{2} \gamma_{3} / \gamma_{4}=\gamma_{1} \gamma_{3} / \gamma_{4}$. We write $\sigma_{1} \| \sigma_{2}$ if stack signatures $\sigma_{1}$ and $\sigma_{2}$ are strictly compatible.

By introducing an element $T$ and extending the definition $\cdot$ as follows, $\left\langle\left(\Gamma^{*} \times \Gamma^{*}\right) \cup\{\top\}\right.$, $\cdot, \epsilon / \epsilon\rangle$ forms a monoid. The proof of the associativity of $\cdot$ appears in Appendix A. We write $\mathcal{M}_{\Gamma}$ for this monoid.

$$
\begin{array}{ll}
\top \cdot \sigma=\sigma \cdot \top=\top & \text { for } \sigma \in \mathcal{M}_{\Gamma} \\
w_{1} / w_{1}^{\prime} \cdot w_{2} / w_{2}^{\prime}=\top & \text { if } w_{1} / w_{1}^{\prime} \text { and } w_{2} / w_{2}^{\prime} \text { are not compatible }
\end{array}
$$

By relaxing the use of terminology, we call an element of $\mathcal{M}_{\Gamma}$ a stack signature and an element of the form $w / w^{\prime}$ a proper stack signature.

The following isomorphism is used to relate automata and pushdown systems. It is clear from $w_{1} / \epsilon \cdot w_{2} / \epsilon=w_{1} w_{2} / \epsilon$.

Proposition 3.2. The set $\left\{w / \epsilon \mid w \in \Gamma^{*}\right\}$ is a submonoid of $\mathcal{M}_{\Gamma}$. Furthermore, it is isomorphic to $\Gamma^{*}$ by the function projecting $w$ from $w / \epsilon$.

We also introduce a partial order on stack signatures: a transition that pops $w_{1}$ and pushes $w_{2}$ can be considered as one that pops $w_{1} w$ and pushes $w_{2} w$ for any $w \in \Gamma^{*}$.

Definition 3.3. A partial order $\leq$ on stack signatures is defined by $w_{1} / w_{2} \leq w_{1} w / w_{2} w$ for $w_{1}, w_{2}, w \in \Gamma^{*}$ and $\sigma \leq \top$ for any stack signature $\sigma$.

It is clear that $\left(\Gamma^{*} \times \Gamma^{*}\right) \cup\{T\}$ is a join-semilattice. This partial order is compatible with the binary operation $\because$ if $\sigma_{1} \leq \sigma_{1}^{\prime}$ and $\sigma_{2} \leq \sigma_{2}^{\prime}$, then $\sigma_{1} \cdot \sigma_{2} \leq \sigma_{1}^{\prime} \cdot \sigma_{2}^{\prime}$ (Lemma A.3 in the appendix). Thus, the monoid of stack signatures is an ordered monoid ${ }^{4}$. With this order, the compatibility of stack signatures can be understood by the strict compatibility.

Lemma 3.4. Two stack signatures $\sigma_{1}$ and $\sigma_{2}$ are compatible if and only if one of the following holds.

\footnotetext{
${ }^{3}$ The proof script can be found at http://www.is.titech.ac.jp/ minamide/stacksig.tar.gz

${ }^{4} \mathrm{~A}$ monoid is ordered when it is equipped with a compatible partial order.
} 
- $\sigma_{1} \leq \sigma_{1}^{\prime}$ and $\sigma_{1}^{\prime} \| \sigma_{2}$ for some $\sigma_{1}^{\prime}$.

- $\sigma_{2} \leq \sigma_{2}^{\prime}$ and $\sigma_{1} \| \sigma_{2}^{\prime}$ for some $\sigma_{2}^{\prime}$.

For example, $\gamma_{1} \gamma_{2} / \gamma_{3}$ and $\gamma_{3} \gamma_{4} / \gamma_{5}$ are compatible because $\gamma_{1} \gamma_{2} / \gamma_{3} \leq \gamma_{1} \gamma_{2} \gamma_{4} / \gamma_{3} \gamma_{4}$ and $\gamma_{1} \gamma_{2} \gamma_{4} / \gamma_{3} \gamma_{4} \| \gamma_{3} \gamma_{4} / \gamma_{5}$. Then, · on compatible stack signatures can also be understood by · on strictly compatible stack signatures.

\section{Lemma 3.5.}

- If $\sigma_{1} \leq \sigma_{1}^{\prime}$ and $\sigma_{1}^{\prime} \| \sigma_{2}$, then $\sigma_{1} \cdot \sigma_{2}=\sigma_{1}^{\prime} \cdot \sigma_{2}$.

- If $\sigma_{2} \leq \sigma_{2}^{\prime}$ and $\sigma_{1} \| \sigma_{2}^{\prime}$, then $\sigma_{1} \cdot \sigma_{2}=\sigma_{1} \cdot \sigma_{2}^{\prime}$.

Furthermore, we can construct an idempotent semiring by introducing the bottom element $\perp$ and extending · for $\perp$ as follows.

$$
\perp \cdot x=x \cdot \perp=\perp \quad \text { for all } x \in\left(\Gamma^{*} \times \Gamma^{*}\right) \cup\{\top, \perp\}
$$

Proposition 3.6. Let $S=\left(\Gamma^{*} \times \Gamma^{*}\right) \cup\{\top, \perp\}$. $\langle S, \sqcup, \cdot, \perp, \epsilon / \epsilon\rangle$ forms an idempotent semiring.

The distributivity of $\cdot$ over $\sqcup$ is proved in Lemma A.5. This semiring is not bounded because $\epsilon / \epsilon \leq \gamma / \gamma \leq \gamma \gamma / \gamma \gamma \leq \cdots$.

\section{SEmirings IndeXed By A Monoid}

We introduce a semiring indexed by a monoid, which is a typed algebraic structure where a type is an element of a monoid. Weighted pushdown systems are generalized by taking this structure as the weight domain in the next section.

Definition 4.1. Let $\mathcal{M}=\left\langle M, \cdot 1_{\mathcal{M}}\right\rangle$ be a monoid. An indexed semiring $\mathcal{S}$ over $\mathcal{M}$ is a structure $\left\langle\left\{D_{m}\right\},\left\{\oplus_{m}\right\},\left\{\otimes_{m_{1}, m_{2}}\right\},\left\{0_{m}\right\}, 1\right\rangle$ such that

- $D_{m}$ is a set for each $m \in M$.

- $\left\langle D_{m}, \oplus_{m}, 0_{m}\right\rangle$ is a commutative monoid for $m \in M$.

- $\otimes_{m_{1}, m_{2}}$ is an associative binary operation of type $D_{m_{1}} \times D_{m_{2}} \rightarrow D_{m_{1} m_{2}}$ for $m_{1}, m_{2} \in M$.

$$
\left(a \otimes_{m_{1}, m_{2}} b\right) \otimes_{m_{1} m_{2}, m_{3}} c=a \otimes_{m_{1}, m_{2} m_{3}}\left(b \otimes_{m_{2}, m_{3}} c\right)
$$

- $1 \in D_{1_{\mathcal{M}}}$ is a neutral element of $\otimes_{m, m^{\prime}}: a \otimes_{m, 1_{\mathcal{M}}} 1=1 \otimes_{1_{\mathcal{M}}, m} a=a$.

- $\otimes_{m_{1}, m_{2}}$ distributes over $\oplus_{m}$.

$$
\begin{aligned}
& \left(a \oplus_{m_{1}} b\right) \otimes_{m_{1}, m_{2}} c=\left(a \otimes_{m_{1}, m_{2}} c\right) \oplus_{m_{1} m_{2}}\left(b \otimes_{m_{1}, m_{2}} c\right) \\
& a \otimes_{m_{1}, m_{2}}\left(b \oplus_{m_{2}} c\right)=\left(a \otimes_{m_{1}, m_{2}} b\right) \oplus_{m_{1} m_{2}}\left(a \otimes_{m_{1}, m_{2}} c\right)
\end{aligned}
$$

- $0_{m}$ is an annihilator with respect to $\otimes_{m, m^{\prime}}$.

$$
0_{m_{1}} \otimes_{m_{1}, m_{2}} a=0_{m_{1} m_{2}}=b \otimes_{m_{1}, m_{2}} 0_{m_{2}}
$$

We call $\mathcal{S}$ an idempotent indexed semiring if $\mathcal{S}$ is an indexed semiring where $\oplus_{m}$ is idempotent for all $m \in M$. We introduce partial orders $\sqsubseteq_{m}$ defined by $a \sqsubseteq_{m} b$ iff $a \oplus_{m} b=b$. From distributivity of $\otimes$, it is clear that $\otimes$ is monotonic with respect to $\sqsubseteq m$. If we ignore the monoid structure of each $D_{m}$, this structure corresponds to a lax monoidal functor $F: \mathcal{M} \rightarrow($ Set, $\times,\{*\})$ in category theory. 
Example 4.2. Matrices over a semiring have a similar structure, but are indexed by a subgroup instead of a monoid. Let us consider $m \times n$ matrices over an arbitrary semiring. We write $\langle m, n\rangle$ for the dimensions of $m \times n$ matrices. Then, the set of dimensions forms a subgroup by introducing $T$ and defining the binary operation $\cdot$ as follows.

$$
\left\langle m_{1}, n_{1}\right\rangle \cdot\left\langle m_{2}, n_{2}\right\rangle= \begin{cases}\left\langle m_{1}, n_{2}\right\rangle & \text { if } n_{1}=m_{2} \\ \top & \text { otherwise }\end{cases}
$$

Let $D_{\langle m, n\rangle}$ be the set of $m \times n$ matrices. Then, $D_{\langle m, n\rangle}$ with matrix addition and multiplication forms a semiring indexed by the subgroup of dimensions where $D_{\top}$ is defined as a singleton. For boolean matrices, the indexed semiring is idempotent since the addition of boolean matrices is idempotent.

The following proposition is used later to consider a semiring indexed by a submonoid of the stack signatures. The conditions of an indexed semiring carry over to the substructure.

Proposition 4.3. Let $\mathcal{M}=\left\langle M, \cdot 1_{\mathcal{M}}\right\rangle$ be a monoid and $\mathcal{S}$ a semiring indexed by $\mathcal{M}$. If $\mathcal{M}^{\prime}$ is a submonoid of $\mathcal{M}$, then the restriction of $\mathcal{S}$ on $\mathcal{M}^{\prime}$ is a semiring indexed by $\mathcal{M}^{\prime}$.

The notion of weighted automata can be extended for an indexed semiring over the monoid $\Gamma^{*}$ in the straightforward manner.

Definition 4.4. Let $\mathcal{S}$ be an idempotent semiring $\left\langle\left\{D_{w}\right\},\left\{\oplus_{w}\right\},\left\{\otimes_{w_{1}, w_{2}}\right\},\left\{0_{w}\right\}, 1\right\rangle$ indexed by $\Gamma^{*}$. A weighted automaton $\mathcal{A}$ over $\mathcal{S}$ is a structure $\langle\Gamma, Q, E\rangle$ where $Q$ is a finite set of states, and $E: Q \times \Gamma \times Q \rightarrow \bigcup_{\gamma \in \Gamma} D_{\gamma}$ is a set of transition rules assigning a weight such that $E\left(\left\langle q, \gamma, q^{\prime}\right\rangle\right) \in D_{\gamma}$.

The definition of the transition relation is revised as follows. The only revision is that we apply indexed $\otimes_{w, w^{\prime}}$ to combine two transitions for $w$ and $w^{\prime}$.

- $q \stackrel{\epsilon \mid 1}{\longrightarrow} q$ for any $q \in Q$.

- $q \stackrel{\gamma \mid a}{\longrightarrow} q^{\prime}$ if $a=E\left(\left\langle q, \gamma, q^{\prime}\right\rangle\right)$.

- $q \stackrel{w w^{\prime} \mid a \otimes_{w, w^{\prime}} b}{\longrightarrow} q^{\prime}$ if $q \stackrel{w \mid a}{\longrightarrow} q^{\prime \prime}$ and $q^{\prime \prime} \stackrel{w^{\prime} \mid b}{\longrightarrow} q^{\prime}$.

\section{Weighted Pushdown Systems over an Indexed Semiring and Their REACHABILITY ANALYSIS}

We introduce weighted pushdown systems over a semiring indexed by the monoid of stack signatures. The (generalized) reachability analysis of weighted pushdown systems is refined to those over an indexed semiring and the boundedness is relaxed to the local boundedness. We also show that it is possible to construct an ordinary semiring from an indexed semiring, but the obtained semiring is not bounded.

5.1. Weighted Pushdown Systems over an Indexed Semiring. We basically consider pushdown systems over a stack alphabet $\Gamma$ as automata over the monoid of stack signatures $\mathcal{M}_{\Gamma}$. However, to clarify our presentation we introduce the definition of weighted pushdown systems independently. Weight domains $D_{\sigma}$ are indexed by a stack signature $\sigma$ and forms an indexed semiring over $\mathcal{M}_{\Gamma}$. 
Definition 5.1. Let $\mathcal{S}=\left\langle\left\{D_{\sigma}\right\},\left\{\oplus_{\sigma}\right\},\left\{\otimes_{\sigma_{1}, \sigma_{2}}\right\},\left\{0_{\sigma}\right\}, 1\right\rangle$ be a semiring indexed by $\mathcal{M}_{\Gamma}$. A weighted pushdown system $\mathcal{P}$ over $\mathcal{S}$ is a structure $\langle P, \Gamma, \Delta\rangle$ where $P$ is a finite set of states, $\Gamma$ is a stack alphabet, and $\Delta \subseteq P \times \Gamma \times P \times \Gamma^{*} \times \bigcup_{\gamma \in \Gamma, w \in \Gamma^{*}} D_{\gamma / w}$ is a finite set of transitions such that $a \in D_{\gamma / w}$ for $\left\langle p, \gamma, p^{\prime}, w, a\right\rangle \in \Delta$.

A configuration of a pushdown system $\mathcal{P}$ is a pair $\langle p, w\rangle$ for $p \in P$ and $w \in \Gamma^{*}$. We write $\langle p, \gamma\rangle \stackrel{a}{\hookrightarrow}\left\langle p^{\prime}, w\right\rangle$ if $\left\langle p, \gamma, p^{\prime}, w, a\right\rangle \in \Delta$.

We consider pushdown systems as automata over stack signatures and define the translation relation as follows:

- $p \stackrel{\epsilon / \epsilon \mid 1}{\Longrightarrow} p$.

- $p \stackrel{\gamma / w \mid a}{\longrightarrow} p^{\prime}$ if $\langle p, \gamma\rangle \stackrel{a}{\hookrightarrow}\left\langle p^{\prime}, w\right\rangle$.

- $p \stackrel{\sigma_{1} \cdot \sigma_{2} \mid a}{\Longrightarrow} p^{\prime}$ if $p \stackrel{\sigma_{1} \mid a_{1}}{\Longrightarrow} p^{\prime \prime}, p^{\prime \prime} \stackrel{\sigma_{2} \mid a_{2}}{\Longrightarrow} p^{\prime}, a=a_{1} \otimes_{\sigma_{1}, \sigma_{2}} a_{2}$ and $\sigma_{1} \cdot \sigma_{2} \neq \mathrm{T}$.

Then, it is clear that $a \in D_{\sigma}$ if $p \stackrel{\sigma \mid a}{\Longrightarrow} p^{\prime}$.

Traditionally, the transition relation on a pushdown system is defined as a relation between configurations. To introduce such a definition, we need to extend an indexed semiring with an additional operation.

Definition 5.2. Let $\mathcal{M}$ be an ordered monoid with partial order $\leq$. By an indexed semiring over $\mathcal{M}$ we shall mean an indexed semiring $\mathcal{S}$ over $\mathcal{M}$ on which there is a family of conversion functions $\uparrow_{m, m^{\prime}}: D_{m} \rightarrow D_{m^{\prime}}$ indexed by pairs of monoid elements $m \leq m^{\prime}$ such that

(1) $\uparrow_{m, m}=\mathrm{id}$.

(2) $\uparrow_{m, m^{\prime \prime}}=\uparrow_{m^{\prime}, m^{\prime \prime}} \circ \uparrow_{m, m^{\prime}}$ for all $m \leq m^{\prime} \leq m^{\prime \prime}$.

(3) $\uparrow_{m, m^{\prime}}\left(0_{m}\right)=0_{m^{\prime}}$ and $\uparrow_{m, m^{\prime}}\left(a \oplus_{m} b\right)=\uparrow_{m, m^{\prime}}(a) \oplus_{m^{\prime}} \uparrow_{m, m^{\prime}}(b)$.

(4) $\uparrow_{m_{1} m_{2}, m_{1}^{\prime} m_{2}^{\prime}}\left(a \otimes_{m_{1}, m_{2}} b\right)=\uparrow_{m_{1}, m_{1}^{\prime}}(a) \otimes_{m_{1}^{\prime}, m_{2}^{\prime}} \uparrow_{m_{2}, m_{2}^{\prime}}(b)$ for all $m_{1} \leq m_{1}^{\prime}$ and $m_{2} \leq m_{2}^{\prime}$.

Example 5.3. The structure $\mathcal{S}=\left\langle\left\{D_{\sigma}\right\},\left\{\oplus_{\sigma}\right\},\left\{\otimes_{\sigma, \sigma_{2}}\right\},\left\{0_{\sigma}\right\}, 0\right\rangle$ forms a semiring indexed by the ordered monoid of stack signatures.

- $D_{w / w^{\prime}}=\mathbb{N} \geq \max \left(|w|,\left|w^{\prime}\right|\right) \cup\{\infty\}$ and $D_{\top}=\{\infty\}$ where $\mathbb{N} \geq i=\{j \in \mathbb{N} \mid j \geq i\}$.

- $a \oplus_{\sigma} b=\min (a, b)$ and $0_{\sigma}=\infty$.

- $\otimes_{\sigma_{1}, \sigma_{2}}$ is defined for compatible $\sigma_{1}$ and $\sigma_{2}$ as follows.

$$
a \otimes_{w_{1} / w_{1}^{\prime}, w_{2} / w_{2}^{\prime}} b= \begin{cases}\max \left(\left|w_{2}\right|-\left|w_{1}^{\prime}\right|+a, b\right) & \text { if }\left|w_{1}^{\prime}\right| \leq\left|w_{2}\right| \\ \max \left(a,\left|w_{1}^{\prime}\right|-\left|w_{2}\right|+b\right) & \text { if }\left|w_{2}\right| \leq\left|w_{1}^{\prime}\right|\end{cases}
$$

- The conversion functions are defined by $\uparrow_{w_{1} / w_{1}^{\prime}, w_{1} w / w_{2}^{\prime} w}(a)=a+|w|$.

It is shown in Example 6.5 that the structure $\mathcal{S}$ really satisfies the conditions of indexed semirings through the construction introduced in Section 6. This indexed semiring is used to compute the minimum height of transitions between two configurations of a pushdown system in Example 5.7.

For an indexed semiring over the ordered monoid $\mathcal{M}_{\Gamma}$, we write $\uparrow_{w}$ for $\uparrow_{w_{1} / w_{2}, w_{1} w / w_{2} w}$ if $w_{1}$ and $w_{2}$ are clear from the context. Then, the standard definition of the transition relation of a weighted pushdown system is given as follows.

- $\langle p, w\rangle \stackrel{\uparrow w(1)}{\longrightarrow}\langle p, w\rangle$.

- $\left\langle p, \gamma w^{\prime}\right\rangle \stackrel{\uparrow_{w^{\prime}}(a)}{\Longrightarrow}\left\langle p^{\prime}, w w^{\prime}\right\rangle$ if $\langle p, \gamma\rangle \stackrel{a}{\hookrightarrow}\left\langle p^{\prime}, w\right\rangle$.

- $\langle p, w\rangle \stackrel{a}{\Rightarrow}\left\langle p^{\prime}, w^{\prime}\right\rangle$ if $\langle p, w\rangle \stackrel{a_{1}}{\Longrightarrow}\left\langle p^{\prime \prime}, w^{\prime \prime}\right\rangle,\left\langle p^{\prime \prime}, w^{\prime \prime}\right\rangle \stackrel{a_{2}}{\Longrightarrow}\left\langle p^{\prime}, w^{\prime}\right\rangle$, and $a=a_{1} \otimes_{w / w^{\prime \prime}, w^{\prime \prime} / w^{\prime}} a_{2}$. 
Then, these two definitions of transition relations are equivalent in the following sense. As a special case of this proposition, we have $\langle p, w\rangle \stackrel{a}{\Rightarrow}\left\langle p^{\prime}, \epsilon\right\rangle$ iff $p \stackrel{w / \epsilon \mid a}{\Longrightarrow} p^{\prime}$.

Proposition 5.4. If $\langle p, w\rangle \stackrel{a}{\Rightarrow}\left\langle p^{\prime}, w^{\prime}\right\rangle$, then there exist $\sigma$ and $a^{\prime}$ such that $\sigma \leq w / w^{\prime}$, $p \stackrel{\sigma \mid a^{\prime}}{\Longrightarrow} p^{\prime}$, and $a=\uparrow_{\sigma, w / w^{\prime}}\left(a^{\prime}\right)$. Conversely, if $p \stackrel{\sigma \mid a^{\prime}}{\Longrightarrow} p^{\prime}$, then $\langle p, w\rangle \stackrel{\uparrow_{\sigma, w / w^{\prime}}\left(a^{\prime}\right)}{\Longrightarrow}\left\langle p^{\prime}, w^{\prime}\right\rangle$ for all $\sigma \leq w / w^{\prime}$.

Proof. We prove the first direction by induction on the derivation of $\langle p, w\rangle \stackrel{a}{\Rightarrow}\left\langle p^{\prime}, w^{\prime}\right\rangle$.

Case: $\langle p, w\rangle \stackrel{\uparrow_{w}(1)}{\Longrightarrow}\langle p, w\rangle$. We have $p \stackrel{\epsilon / \epsilon \mid 1}{\Longrightarrow} p, \epsilon / \epsilon \leq w / w$, and $\uparrow_{w}(1)=\uparrow_{\epsilon / \epsilon, w / w}(1)$.

Case: $\left\langle p, \gamma w^{\prime}\right\rangle \stackrel{\uparrow_{w^{\prime}}(a)}{\Longrightarrow}\left\langle p^{\prime}, w w^{\prime}\right\rangle$. We have $p \stackrel{\gamma / w \mid a}{\Longrightarrow} p^{\prime}$ and $\gamma / w \leq \gamma w^{\prime} / w w^{\prime}$.

Case: $\langle p, w\rangle \stackrel{a}{\Rightarrow}\left\langle p^{\prime}, w^{\prime}\right\rangle$ is obtained from $\langle p, w\rangle \stackrel{a_{1}}{\Longrightarrow}\left\langle p^{\prime \prime}, w^{\prime \prime}\right\rangle,\left\langle p^{\prime \prime}, w^{\prime}\right\rangle \stackrel{a_{2}}{\Rightarrow}\left\langle p^{\prime}, w^{\prime}\right\rangle$, and $a=a_{1} \otimes_{w / w^{\prime \prime}, w^{\prime \prime} / w^{\prime}} a_{2}$. By the induction hypothesis, we have

- $p \stackrel{\sigma_{1} \mid a_{1}^{\prime}}{\Longrightarrow} p^{\prime \prime}, \sigma_{1} \leq w / w^{\prime \prime}$, and $\uparrow_{\sigma_{1}, w / w^{\prime \prime}}\left(a_{1}^{\prime}\right)=a_{1}$,

- $p^{\prime \prime} \stackrel{\sigma_{2} \mid a_{2}^{\prime}}{\Longrightarrow} p^{\prime}, \sigma_{2} \leq w^{\prime \prime} / w^{\prime}$, and $\uparrow_{\sigma_{2}, w^{\prime \prime} / w^{\prime}}\left(a_{2}^{\prime}\right)=a_{2}$.

By monotonicity of $\cdot, \sigma_{1} \cdot \sigma_{2} \leq w / w^{\prime}$ and then $p \stackrel{\sigma_{1} \cdot \sigma_{2} \mid a^{\prime}}{\Longrightarrow} p^{\prime}$ where $a^{\prime}=a_{1}^{\prime} \otimes_{\sigma_{1}, \sigma_{2}} a_{2}^{\prime}$. We also have $\uparrow_{\sigma_{1} \cdot \sigma_{2}, w / w^{\prime}}\left(a_{1}^{\prime} \otimes_{\sigma_{1}, \sigma_{2}} a_{2}^{\prime}\right)=\uparrow_{\sigma_{1}, w / w^{\prime \prime}}\left(a_{1}^{\prime}\right) \otimes_{w / w^{\prime \prime}, w^{\prime \prime} / w^{\prime}} \uparrow_{\sigma_{2}, w^{\prime \prime}} / w^{\prime}\left(a_{2}^{\prime}\right)=a$.

The other direction is proved in a similar manner by induction on the derivation of $p \stackrel{\sigma \mid a^{\prime}}{\Longrightarrow} p^{\prime}$.

5.2. Reachability Analysis. We show that the reachability analysis of weighted pushdown systems by Reps et al. RSJM05 can be generalized for those over an indexed semiring, where we adopt a localized version of the boundedness of a semiring.

Definition 5.5. We say an indexed idempotent semiring over $\mathcal{M}_{\Gamma}$ is locally bounded if $D_{\gamma / \epsilon}$ is bounded for all $\gamma \in \Gamma$.

First, we focus on the (generalized) backward reachability of a configuration with the empty stack and consider the problem that computes the following function:

$$
\delta\left(p, w, p^{\prime}\right)=\bigoplus\left\{a \mid p \stackrel{w / \epsilon \mid a}{\Longrightarrow} p^{\prime}\right\}
$$

where the above addition is the extension of $\oplus_{w / \epsilon}$ for a set. This function is well-defined if the indexed semiring is locally bounded. It is clear from the following equation:

$$
\delta\left(p, \gamma w^{\prime}, p^{\prime}\right)=\bigoplus_{p^{\prime \prime} \in P}\left(\delta\left(p, \gamma, p^{\prime \prime}\right) \otimes_{\gamma / \epsilon, w^{\prime} / \epsilon} \delta\left(p^{\prime \prime}, w^{\prime}, p^{\prime}\right)\right)
$$

where we have $\delta\left(p, \gamma, p^{\prime \prime}\right) \in D_{\gamma / \epsilon}$ for all $p^{\prime \prime} \in P$. Although there are infinitely many transitions of the form $p \stackrel{\gamma / \epsilon \mid a}{\Longrightarrow} p^{\prime \prime}, \delta\left(p, \gamma, p^{\prime \prime}\right)$ is well-defined because $D_{\gamma / \epsilon}$ is bounded.

We generalize the reachability analysis of weighted pushdown automata for those over an indexed semiring. The algorithm is a generalization of the saturation procedure on $\mathcal{P}$-automata BEM97, FWW97, RSJM05.

Let us consider a weighted pushdown system $\mathcal{P}=\langle P, \Gamma, \Delta\rangle$ over a semiring $\mathcal{S}$ indexed by $\mathcal{M}_{\Gamma}$. We apply the procedure to a weighted automaton over the restriction of $\mathcal{S}$ to $\{w / \epsilon \mid$ 
$\left.\left.w \in \Gamma^{*}\right\}\right]^{5}$ and start from $\mathcal{A}_{0}=\left\langle P, \Gamma, E_{0}\right\rangle$, which has no transitions, i.e., $E_{0}\left(\left\langle p, \gamma, p^{\prime}\right\rangle\right)=0_{\gamma / \epsilon}$ for all $p, p^{\prime} \in P$ and $\gamma \in \Gamma$. Then, the weighted automaton $\mathcal{A}_{\text {pre* }}$ representing $\delta_{\mathcal{P}}\left(p, \gamma, p^{\prime}\right)$ can be obtained by applying the saturation rule for weighted pushdown systems to $\mathcal{A}_{0}$ until saturation. The following is the saturation rule of Reps et al. for the backward reachability analysis adapted to our framework [RSJM05].

- If $\langle p, \gamma\rangle \stackrel{a_{1}}{\hookrightarrow}\left\langle p^{\prime}, w\right\rangle$ and $p^{\prime} \stackrel{w \mid a_{2}}{\longrightarrow} p^{\prime \prime}$ in the current automaton, add a transition rule $p \stackrel{\gamma \mid a}{\longrightarrow} p^{\prime \prime}$ to the automaton where $a=a_{1} \otimes_{\gamma / w, w / \epsilon} a_{2}$.

When we add $p \stackrel{\gamma \mid a}{\longrightarrow} p^{\prime \prime}$, if there already exists transition $p \stackrel{\gamma \mid a^{\prime}}{\longrightarrow} p^{\prime \prime}$, then we replace it with $p \stackrel{\gamma \mid a \oplus_{\gamma / \epsilon} a^{\prime}}{\longrightarrow} p^{\prime \prime}$.

Since there are only finitely many (one-step) transitions in $\mathcal{A}_{\mathrm{pre}}$, it is clear that the application of the rule terminates if the indexed semiring is locally bounded.

Theorem 5.6. Let $\mathcal{P}$ be a weighted pushdown system over a locally bounded idempotent semiring indexed by $\mathcal{M}_{\Gamma}$.

- The saturation procedure above terminates.

- Let $\mathcal{A}_{\mathrm{pre}}$ be a weighted automaton obtained by the saturation procedure. Then, we have $p \underset{\mathcal{A}_{\text {pre* }}}{\stackrel{\gamma \mid a}{\longrightarrow}} p^{\prime}$ for $a=\delta_{\mathcal{P}}\left(p, \gamma, p^{\prime}\right)$.

As a corollary, we have $p \underset{\mathcal{A}_{\mathrm{pre}^{*}}}{\stackrel{w \mid a}{\longrightarrow}} p^{\prime}$ for $a=\delta_{\mathcal{P}}\left(p, w, p^{\prime}\right)$. Before the proof of the theorem, we illustrate the saturation procedure by an example.

Example 5.7. The minimum height of transitions between two configurations can be computed by the indexed semiring of Example 5.3. Let $\mathcal{P}=\langle P, \Gamma, \Delta\rangle$ be an ordinary pushdown system. For a computation $\mathcal{C}:\left\langle p_{1}, w_{1}\right\rangle \Longrightarrow\left\langle p_{2}, w_{2}\right\rangle \Longrightarrow \cdots \Longrightarrow\left\langle p_{n}, w_{n}\right\rangle$ of $\mathcal{P}$, the height of $\mathcal{C}$ is defined by height $(\mathcal{C})=\max _{1 \leq i \leq n}\left|w_{i}\right|$. We then consider the minimum height of computations between two configurations.

The minimum height can be determined by the reachability analysis of the weighted pushdown system $\mathcal{P}^{\prime}=\left\langle P, \Gamma, \Delta^{\prime}\right\rangle$ where $\Delta^{\prime}$ is given by: $\left\langle p, \gamma, p^{\prime}, w, \max (1,|w|)\right\rangle \in \Delta^{\prime}$ if $\left\langle p, \gamma, p^{\prime}, w\right\rangle \in \Delta$. Then, we have the following transitions in $\mathcal{P}^{\prime}$.

- For a transition with no real moves, $\langle p, w\rangle \stackrel{\uparrow_{\epsilon / \epsilon, w / w}(0)}{\underset{\mathcal{P}^{\prime}}{\Longrightarrow}}\langle p, w\rangle$ where $\uparrow_{\epsilon / \epsilon, w / w}(0)=|w|$.

- For a one-step transition for $\left\langle p_{1}, \gamma, p_{2}, w\right\rangle \in \Delta$, we have

$$
\left\langle p_{1}, \gamma w^{\prime}\right\rangle \stackrel{\uparrow_{\gamma / w, \gamma w^{\prime} / w w^{\prime}}(\max (1,|w|))}{\mathcal{P}^{\prime}}\left\langle p_{2}, w w^{\prime}\right\rangle
$$

where $\uparrow_{\gamma / w, \gamma w^{\prime} / w w^{\prime}}\left(\max (1,|w|)=\max (1,|w|)+\left|w^{\prime}\right|=\max \left(\left|\gamma w^{\prime}\right|,\left|w w^{\prime}\right|\right)\right.$.

- For $\left\langle p_{1}, w_{1}\right\rangle \stackrel{n_{1}}{\underset{\mathcal{P}^{\prime}}{\Rightarrow}}\left\langle p_{2}, w_{2}\right\rangle$ and $\left\langle p_{2}, w_{2}\right\rangle \stackrel{n_{2}}{\underset{\mathcal{P}^{\prime}}{2}}\left\langle p_{3}, w_{3}\right\rangle$, we have $\left\langle p_{1}, w_{1}\right\rangle \stackrel{\max \left(n_{1}, n_{2}\right)}{=}\left\langle p_{3}, w_{3}\right\rangle$.

Thus, we can compute the minimum height of computations by the reachability analysis of $\mathcal{P}^{\prime}$.

5 The restriction of $\mathcal{S}$ to $\left\{w / \epsilon \mid w \in \Gamma^{*}\right\}$ is a semiring indexed by $\left\{w / \epsilon \mid w \in \Gamma^{*}\right\}$ by Proposition 3.2 and 4.3 


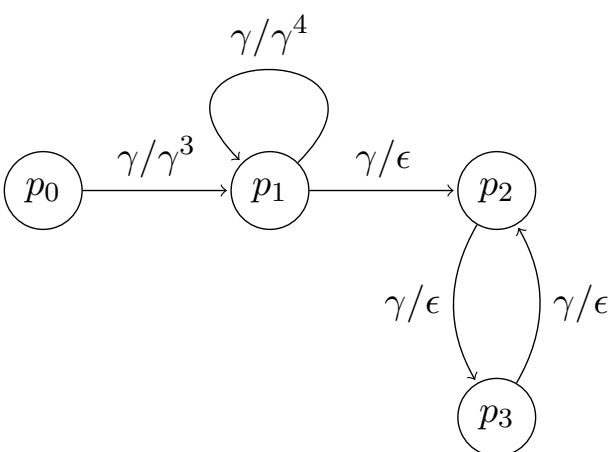

(a)

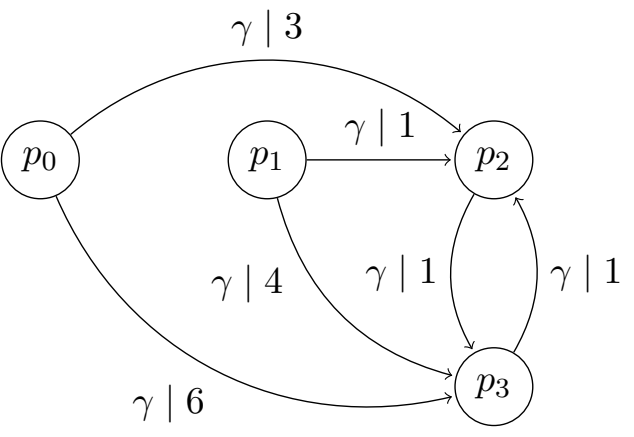

(b)

Figure 1: (a) pushdown system $\mathcal{P}_{\text {ex }}$. (b) weighted automaton $\mathcal{A}_{\text {pre* }}$ of $\mathcal{P}_{\text {ex }}$.

Let us consider the pushdown system $\mathcal{P}_{\text {ex }}$ in Figure 1. $\mathcal{P}_{\text {ex }}$ is designed so that the following holds.

$$
\begin{aligned}
& \left\langle p_{0}, \gamma \gamma^{m}\right\rangle \Longrightarrow\left\langle p_{1}, w\right\rangle \quad \text { iff } \quad w=\gamma^{3 n+m} \text { for some } n>0 \\
& \left\langle p_{1}, w\right\rangle \Longrightarrow\left\langle p_{3}, \epsilon\right\rangle \quad \text { iff } \quad w=\gamma^{2 n} \text { for some } n>0
\end{aligned}
$$

Thus, the minimum height of computations between $\left\langle p_{0}, \gamma\right\rangle$ and $\left\langle p_{3}, \epsilon\right\rangle$ is 6 .

Let us determine this by the reachability analysis of $\mathcal{P}_{\text {ex }}^{\prime}$. We apply the saturation procedure to $\mathcal{P}_{\text {ex }}^{\prime}$.

(1) From $\left\langle p_{1}, \gamma\right\rangle \stackrel{1}{\hookrightarrow}\left\langle p_{2}, \epsilon\right\rangle$ and $p_{2} \stackrel{\epsilon \mid 0}{\longrightarrow} p_{2}$, we add $p_{1} \stackrel{\gamma \mid a_{1}}{\longrightarrow} p_{2}$ where $a_{1}=1 \otimes_{\gamma / \epsilon, \epsilon / \epsilon} 0=$ $\max (1,0)=1$. Similarly, we add $p_{2} \stackrel{\gamma \mid 1}{\longrightarrow} p_{3}$ and $p_{3} \stackrel{\gamma \mid 1}{\longrightarrow} p_{2}$.

(2) From $p_{1} \stackrel{\gamma \mid 1}{\longrightarrow} p_{2}$ and $p_{2} \stackrel{\gamma \mid 1}{\longrightarrow} p_{3}$, we have $p_{1} \stackrel{\gamma^{2} \mid a_{2}}{\longrightarrow} p_{3}$ where $a_{2}=1 \otimes_{\gamma / \epsilon, \gamma / \epsilon} 1=$ $\max (1+1,1)=2$. Similarly, we have $p_{1} \stackrel{\gamma^{3} \mid 3}{\longrightarrow} p_{2}$.

Then, from $\left\langle p_{0}, \gamma\right\rangle \stackrel{3}{\hookrightarrow}\left\langle p_{1}, \gamma / \gamma^{3}\right\rangle$ and $p_{1} \stackrel{\gamma^{3} \mid 3}{\longrightarrow} p_{2}$, we add $p_{0} \stackrel{\gamma \mid 3}{\longrightarrow} p_{2}$.

(3) The other two transitions are added in the same manner.

The transition $p_{0} \stackrel{\gamma \mid 6}{\longrightarrow} p_{3}$ in $\mathcal{A}_{\text {pre* }}$ corresponds to the following computation of $\mathcal{P}_{\text {ex }}$.

$$
\left\langle p_{0}, \gamma\right\rangle \Longrightarrow\left\langle p_{1}, \gamma^{3}\right\rangle \Longrightarrow\left\langle p_{1}, \gamma^{6}\right\rangle \Longrightarrow \cdots \Longrightarrow\left\langle p_{3}, \epsilon\right\rangle
$$

The theorem is proved from the following two lemmas.

Lemma 5.8. If $p \stackrel{w / \epsilon \mid a}{\underset{\mathcal{P}}{\longrightarrow}} p^{\prime}$, then $p \underset{\mathcal{A}_{\mathrm{pre}^{*}}}{\stackrel{w \mid a^{\prime}}{\longrightarrow}} p^{\prime}$ and $a \sqsubseteq_{w / \epsilon} a^{\prime}$ for some $a^{\prime}$.

Proof. If we only consider the transition relation of the form $p \underset{\mathcal{P}}{\stackrel{w / \epsilon \mid}{\Rightarrow}} p^{\prime}$, it has the following equivalent inductive definition.

- $p \stackrel{\epsilon / \epsilon \mid 1}{\Longrightarrow} p$.

- $p \stackrel{\gamma w / \epsilon \mid a}{\longrightarrow} p^{\prime}$ if $\langle p, \gamma\rangle \stackrel{a_{1}}{\hookrightarrow}\left\langle p^{\prime \prime}, w^{\prime}\right\rangle, p^{\prime \prime} \stackrel{w^{\prime} w / \epsilon \mid a_{2}}{=} p^{\prime}$, and $a=a_{1} \otimes_{\gamma / w^{\prime}, w^{\prime} w / \epsilon} a_{2}$.

By induction on the derivation of $p \stackrel{w / \epsilon \mid a}{\stackrel{\mathcal{P}}{\Rightarrow}} p^{\prime}$ in the above form.

Case: $p \stackrel{\epsilon / \epsilon \mid 1}{\Longrightarrow} p$. The claim holds because $p \stackrel{\epsilon \mid 1}{\longrightarrow} p$. 
Case: $p \stackrel{\gamma w_{2} / \epsilon \mid a}{\Longrightarrow} p^{\prime}$ is obtained from $\langle p, \gamma\rangle \stackrel{a_{0}}{\hookrightarrow}\left\langle p^{\prime \prime}, w_{1}\right\rangle, p^{\prime \prime} \stackrel{w_{1} w_{2} / \epsilon \mid a_{3}}{\Longrightarrow} p^{\prime}$, and $a=a_{0} \otimes_{\gamma / w_{1}, w_{1} w_{2} / \epsilon}$ $a_{3}$. By induction hypothesis, $p^{\prime \prime} \stackrel{w_{1} w_{2} \mid a_{3}^{\prime}}{\longrightarrow} p^{\prime}$ and $a_{3} \sqsubseteq_{w_{1} w_{2} / \epsilon} a_{3}^{\prime}$. Then, we have

$$
p^{\prime \prime} \stackrel{w_{1} \mid a_{1}^{\prime}}{\longrightarrow} p^{\prime \prime \prime} \quad p^{\prime \prime \prime} \stackrel{w_{2} \mid a_{2}^{\prime}}{\longrightarrow} p^{\prime}
$$

and $a_{3}^{\prime}=a_{1}^{\prime} \otimes_{w_{1} / \epsilon, w_{2} / \epsilon} a_{2}^{\prime}$ for some $p^{\prime \prime \prime}, a_{1}^{\prime}$, and $a_{2}^{\prime}$.

Let $\mathcal{A}_{\text {pre* }}=\left\langle P, \Gamma, E_{\text {pre* }}\right\rangle$. By construction of $\mathcal{A}_{\text {pre* }}$,

$$
a_{0} \otimes_{\gamma / w_{1}, w_{1} / \epsilon} a_{1}^{\prime} \sqsubseteq_{\gamma / \epsilon} E_{\mathrm{pre}^{*}}\left(\left\langle p, \gamma, p^{\prime \prime \prime}\right\rangle\right)
$$

Hence

and

$$
\begin{array}{rll}
a=a_{0} \otimes_{\gamma / w_{1}, w_{1} w_{2} / \epsilon} a_{3} & \sqsubseteq_{\gamma w_{2} / \epsilon} & a_{0} \otimes_{\gamma / w_{1}, w_{1} w_{2} / \epsilon}\left(a_{1}^{\prime} \otimes_{w_{1} / \epsilon, w_{2} / \epsilon} a_{2}^{\prime}\right) \\
& \sqsubseteq_{\gamma w_{2} / \epsilon} \quad E_{\mathrm{pre}^{*}}\left(\left\langle p, \gamma, p^{\prime \prime \prime}\right\rangle\right) \otimes_{\gamma, w_{2}} a_{2}^{\prime}
\end{array}
$$

$$
p \stackrel{\gamma w_{2} \mid E_{\mathrm{pre}}\left(\left\langle p, \gamma, p^{\prime \prime \prime}\right\rangle\right) \otimes_{\gamma, w_{2}} a_{2}^{\prime}}{\longrightarrow} p^{\prime}
$$

Let $\mathcal{A}_{i+1}$ be a weighted automaton obtained by applying the saturation rule once to $\mathcal{A}_{i}$.

Lemma 5.9. If $p \underset{\mathcal{A}_{i}}{\stackrel{\gamma \mid a}{\longrightarrow}} p^{\prime}$, then $a \sqsubseteq_{\gamma / \epsilon} \delta_{\mathcal{P}}\left(p, \gamma, p^{\prime}\right)$.

Proof. By induction on $i$. For $i=0$, the statement trivially holds because $a=0_{\gamma / \epsilon}$ for $p \underset{\mathcal{A}_{0}}{\stackrel{\gamma \mid a}{\longrightarrow}} p^{\prime}$. By assuming the case for $i$, we show the case for $i+1$. We only consider the case where $p \underset{\mathcal{A}_{i+1}}{\stackrel{\gamma \mid a}{\longrightarrow}} p^{\prime}$ is added by the last application of the saturation rule. Let us assume that $p \underset{\mathcal{A}_{i+1}}{\stackrel{\gamma \mid a}{\longrightarrow}} p^{\prime}$ is added because of $\langle p, \gamma\rangle \stackrel{a_{1}}{\longrightarrow}\left\langle p^{\prime \prime}, w\right\rangle, p^{\prime \prime} \underset{\mathcal{A}_{i}}{\stackrel{w \mid a_{2}}{\longrightarrow}} p^{\prime}, p \underset{\mathcal{A}_{i}}{\stackrel{\gamma \mid a_{0}}{\longrightarrow}} p^{\prime}$, and $a=a_{1} \otimes_{\gamma / w, w / \epsilon} a_{2} \oplus_{\gamma / \epsilon} a_{0}$.

By induction hypothesis, $a_{2} \sqsubseteq_{w / \epsilon} \delta_{\mathcal{P}}\left(p^{\prime \prime}, w, p^{\prime}\right)$ and $a_{0} \sqsubseteq_{\gamma / \epsilon} \delta_{\mathcal{P}}\left(p, \gamma, p^{\prime}\right)$. We also have $a_{1} \otimes_{\gamma / w, w / \epsilon} \delta_{\mathcal{P}}\left(p^{\prime \prime}, w, p^{\prime}\right) \sqsubseteq_{\gamma / \epsilon} \delta_{\mathcal{P}}\left(p, \gamma, p^{\prime}\right)$ from $\langle p, \gamma\rangle \stackrel{a_{1}}{\longrightarrow}\left\langle p^{\prime \prime}, w\right\rangle$. Hence, $a_{1} \otimes_{\gamma / w, w / \epsilon} a_{2} \sqsubseteq_{\gamma / \epsilon}$ $a_{1} \otimes \delta_{\mathcal{P}}\left(p^{\prime \prime}, w, p^{\prime}\right) \sqsubseteq_{\gamma / \epsilon} \delta_{\mathcal{P}}\left(p, \gamma, p^{\prime}\right)$. Thus, $a \sqsubseteq_{\gamma / \epsilon} \delta_{\mathcal{P}}\left(p, \gamma, p^{\prime}\right)$.

5.3. Reachability to a Regular Set of Configurations. In previous works of the reachability analysis of pushdown systems, it is common to consider the reachability problem to a regular set of configurations. For a weighted pushdown automaton over an indexed semiring, this problem must be generalized for a regular set with weight represented by a weighted automaton.

Let us consider an indexed semiring $\mathcal{S}$ over $\mathcal{M}_{\Gamma}$ and a weighted pushdown system $\mathcal{P}$ over $\mathcal{S}$. We also consider a weighted automaton $\mathcal{A}$ over the restriction of $\mathcal{S}$ to $\left\{w / \epsilon \mid w \in \Gamma^{*}\right\}$ with the initial states $q_{0}$ and the set of final states $F$. Without loss of generality, we assume that there are no incoming transitions to $q_{0}$. For a given state $p^{\prime}, \mathcal{A}$ represents the set of configurations $\left\{\left\langle p^{\prime}, w^{\prime}\right\rangle \mid w^{\prime}\right.$ is accepted by $\left.\mathcal{A}\right\}$. Then, the generalized reachability problem 
to the regular set of configurations is to compute the following function ${ }^{6}$

$$
\delta_{\mathcal{P}, \mathcal{A}}\left(p, w, p^{\prime}\right)=\bigoplus_{q \in F}\left\{a \otimes_{\sigma, w^{\prime} / \epsilon} a^{\prime} \mid p \underset{\mathcal{P}}{\stackrel{\sigma \mid a}{\Longrightarrow}} p^{\prime}, q_{0} \underset{\mathcal{A}}{\stackrel{w^{\prime} \mid a^{\prime}}{\longrightarrow}} q, \text { and } \sigma \cdot w^{\prime} / \epsilon=w / \epsilon\right\}
$$

This function can be computed by applying the saturation procedure to the pushdown system $\mathcal{P}^{\prime}$ obtained by combining $\mathcal{P}$ and $\mathcal{A}$ with the identification of $p^{\prime}$ and $q_{0}$. This corresponds to the saturation procedure using $\mathcal{P}$-automata.

The condition $\sigma \cdot w^{\prime} / \epsilon=w / \epsilon$ above is equivalent to $\sigma \leq w / w^{\prime}$. Furthermore, if the indexed semiring is equipped with the conversion functions $\uparrow_{\sigma_{1}, \sigma_{2}}$, we have the following.

$$
\begin{aligned}
\delta_{\mathcal{P}, \mathcal{A}}\left(p, w, p^{\prime}\right) & =\bigoplus_{q \in F}\left\{a \otimes_{\sigma, w^{\prime} / \epsilon} a^{\prime} \mid p \underset{\mathcal{P}}{\stackrel{\sigma \mid a}{\longrightarrow}} p^{\prime}, q_{0} \underset{\mathcal{A}}{\stackrel{w^{\prime} \mid a^{\prime}}{\longrightarrow}} q, \text { and } \sigma \cdot w^{\prime} / \epsilon=w / \epsilon\right\} \\
& =\bigoplus_{q \in F}\left\{\uparrow_{\sigma, w / w^{\prime}}(a) \otimes_{w / w^{\prime}, w^{\prime} / \epsilon} a^{\prime} \mid p \underset{\mathcal{P}}{\stackrel{\sigma \mid a}{\longrightarrow}} p^{\prime}, q_{0} \underset{w^{\prime} \mid a^{\prime}}{\mathcal{A}} q, \text { and } \sigma \leq w / w^{\prime}\right\}
\end{aligned}
$$

(by Definition $5.2(3)$ )

$$
=\bigoplus_{q \in F}\left\{a \otimes_{w / w^{\prime}, w^{\prime} / \epsilon} a^{\prime} \mid\langle p, w\rangle \underset{\mathcal{P}}{\stackrel{a}{\Rightarrow}}\left\langle p^{\prime}, w^{\prime}\right\rangle \text { and } q_{0} \frac{w^{\prime} \mid a^{\prime}}{\mathcal{A}} q\right\}
$$

(by Proposition 5.4)

The reason why we need to consider a weighted automaton $\mathcal{A}$ instead of just an automaton is that $D_{w / \epsilon}$ does not have a neutral element on $\otimes$ in general. Thus, we need to consider $a^{\prime}$ above.

\subsection{Constructing a Semiring from an indexed Semiring over Stack Signatures.} We show that an ordinary semiring can be constructed from a semiring indexed by stack signatures. However, the semiring obtained by the construction is not bounded even for a locally bounded indexed semiring. Thus, the standard framework of the reachability analysis of weighted pushdown systems cannot guarantee termination of the saturation procedure. Although a similar construction appears in [Suw09], the definition of $\oplus$ differs from ours and his construction fails to satisfy the distributivity of $\otimes$ over $\oplus$.

Let $\mathcal{S}=\left\langle\left\{D_{\sigma}\right\},\left\{\oplus_{\sigma}\right\},\left\{\otimes_{\sigma_{1}, \sigma_{2}}\right\},\left\{0_{\sigma}\right\}, 1_{\mathcal{S}}, \uparrow_{\sigma, \sigma^{\prime}}\right\rangle$ be a semiring indexed by the ordered monoid $\mathcal{M}_{\Gamma}$. Then, we define a structure $\langle D, \oplus, \otimes, \perp, 1\rangle$ as follows.

- $D=\bigcup_{\sigma \in \mathcal{M}_{\Gamma}}\left\{\langle\sigma, a\rangle \mid a \in D_{\sigma}\right\} \cup\{\perp\}$.

- 1 is $\left\langle\epsilon / \epsilon, 1_{\mathcal{S}}\right\rangle$.

- $\oplus$ is defined by $\perp \oplus x=x=x \oplus \perp$ for all $x \in D$ and

$$
\left\langle\sigma_{1}, a\right\rangle \oplus\left\langle\sigma_{2}, b\right\rangle=\left\langle\sigma_{1} \sqcup \sigma_{2}, \uparrow_{\sigma_{1}, \sigma_{1} \sqcup \sigma_{2}}(a) \oplus_{\sigma_{1} \sqcup \sigma_{2}} \uparrow_{\sigma_{2}, \sigma_{1} \sqcup \sigma_{2}}(b)\right\rangle .
$$

- $\otimes$ is defined by $\left\langle\sigma_{1}, a\right\rangle \otimes\left\langle\sigma_{2}, b\right\rangle=\left\langle\sigma_{1} \cdot \sigma_{2}, a \otimes_{\sigma_{1}, \sigma_{2}} b\right\rangle$ and $x \otimes \perp=\perp=\perp \otimes x$ for all $x \in D$.

Theorem 5.10. $\langle D, \oplus, \otimes, \perp, 1\rangle$ forms a semiring.

Proof. We show the associativity of $\oplus$ and the distributivity of $\otimes$ over $\oplus$.

\footnotetext{
${ }^{6}$ For simplicity, we consider the set of configurations whose state is a fixed $p^{\prime}$. It is easy to extend the discussion for the general case.
} 
- Associativity of $\oplus$. Let $\sigma=\sigma_{1} \sqcup \sigma_{2} \sqcup \sigma_{3}$.

$$
\begin{aligned}
\left(\left\langle\sigma_{1}, a\right\rangle \oplus\left\langle\sigma_{2}, b\right\rangle\right) \oplus\left\langle\sigma_{3}, c\right\rangle & =\left\langle\sigma_{1} \sqcup \sigma_{2}, \uparrow \sigma_{1}, \sigma_{1} \sqcup \sigma_{2}(a) \oplus_{\sigma_{1} \sqcup \sigma_{2} \uparrow \sigma_{2}, \sigma_{1} \sqcup \sigma_{2}}(b)\right\rangle \oplus\left\langle\sigma_{3}, c\right\rangle \\
& =\left\langle\sigma, \uparrow \sigma_{1}, \sigma(a) \oplus_{\sigma} \uparrow \sigma_{2}, \sigma\right. \\
& =\left\langle\sigma_{1}, a\right\rangle \oplus \oplus_{\sigma} \uparrow \sigma_{3}, \sigma \\
& (b)\rangle
\end{aligned}
$$

- $\otimes$ distributes over $\oplus$. Let $\sigma=\sigma_{1} \cdot \sigma_{3} \sqcup \sigma_{2} \cdot \sigma_{3}$.

$$
\begin{aligned}
\left(\left\langle\sigma_{1}, a\right\rangle \oplus\left\langle\sigma_{2}, b\right\rangle\right) \otimes\left\langle\sigma_{3}, c\right\rangle & =\left\langle\sigma_{1} \sqcup \sigma_{2}, \uparrow \sigma_{1}, \sigma_{1} \sqcup \sigma_{2}\right. \\
& \left.\left.=\langle a) \oplus_{\sigma_{1}} \sqcup \sigma_{2} \uparrow \sigma_{\sigma_{2}, \sigma_{1}} \sqcup \sigma_{2}(b)\right\rangle \sigma_{1} \sqcup \sigma_{2}(a) \otimes_{\sigma_{1} \sqcup \sigma_{2}, \sigma_{3}} c \oplus_{\sigma} \uparrow \sigma_{\sigma_{2}, \sigma_{1} \sqcup \sigma_{2}}(b) \otimes_{\sigma_{1} \sqcup \sigma_{2}, \sigma_{3}} c\right\rangle \\
& =\left\langle\sigma, \uparrow \sigma_{1} \sigma_{3}, \sigma\right. \\
& =\left\langle a \otimes_{\sigma_{1}, \sigma_{3}} c\right) \oplus_{\sigma} \uparrow \sigma_{2} \sigma_{3}, \sigma \\
& \left.\left(b \otimes_{\sigma_{2}, \sigma_{3}} c\right)\right\rangle \\
& =\left(\left\langle\sigma_{1} \cdot \sigma_{3}, a \otimes_{\sigma_{1}, \sigma_{3}} c\right\rangle \oplus\left\langle\sigma_{2} \cdot \sigma_{3}, b \otimes_{\sigma_{2}, \sigma_{3}} c\right\rangle\right.
\end{aligned}
$$

The construction also works for any semiring indexed by an ordered monoid $\mathcal{M}$ if $\mathcal{M}$ has the join operation $\sqcup$.

Suwimonteerabuth did not consider the partial order on stack signatures and defined the addition of the semiring $\oplus^{\prime}$ in the following manner [Suw09]:

$$
\left\langle\sigma_{1}, a\right\rangle \oplus^{\prime}\left\langle\sigma_{2}, b\right\rangle= \begin{cases}\left\langle\sigma_{1}, a \oplus_{\sigma_{1}} b\right\rangle & \text { if } \sigma_{1}=\sigma_{2} \\ (\top, \bullet) & \text { otherwise }\end{cases}
$$

where we assume $D_{\top}=\{\bullet\}$. However, $\otimes$ does not distribute over $\oplus^{\prime}$, and thus his construction fails to form a semiring.

$$
\begin{aligned}
\left(\langle\epsilon / \epsilon, a\rangle \oplus^{\prime}\langle\gamma / \gamma, b\rangle\right) \otimes\langle\gamma / \gamma, c\rangle & =\langle\top, \bullet\rangle \otimes\langle\gamma / \gamma, c\rangle=\langle\top, \bullet\rangle \\
(\langle\epsilon / \epsilon, a\rangle \otimes\langle\gamma / \gamma, c\rangle) \oplus^{\prime}(\langle\gamma / \gamma, b\rangle \otimes\langle\gamma / \gamma, c\rangle) & \\
& =\left\langle\gamma / \gamma, a \otimes_{\epsilon / \epsilon, \gamma / \gamma} c\right\rangle \oplus^{\prime}\left\langle\gamma / \gamma, b \otimes_{\gamma / \gamma, \gamma / \gamma} c\right\rangle \\
& =\left\langle\gamma / \gamma, a \otimes_{\epsilon / \epsilon, \gamma / \gamma} c \oplus_{\gamma / \gamma} b \otimes_{\gamma / \gamma, \gamma / \gamma} c\right\rangle
\end{aligned}
$$

It should be noted that the semiring constructed in Theorem 5.10 is not bounded as the following sequence shows.

$$
\langle\epsilon / \epsilon, a\rangle \sqsubset\left\langle\gamma / \gamma, \uparrow_{\gamma}(a)\right\rangle \sqsubset\left\langle\gamma \gamma / \gamma \gamma, \uparrow_{\gamma \gamma}(a)\right\rangle \sqsubset \cdots
$$

This is one of the reasons why we refine the formulation of the reachability analysis of weighted pushdown systems in this paper.

The semiring constructed in Theorem 5.10 actually has the structure of a graded semiring. Although a graded structure is usually defined for rings [Lan02, we apply it to semirings. A graded semiring $\langle D, \oplus, \times, 1,0\rangle$ over $\mathcal{M}$ is a semiring where $D=\biguplus_{m \in \mathcal{M}} D_{m}$, $D_{m}$ is a commutative monoid, and $D_{m} D_{m^{\prime}} \subseteq D_{m m^{\prime}}$ for all $m, m^{\prime} \in \mathcal{M}$. It is clear that the semiring in Theorem 5.10 is a graded semiring over $\mathcal{M}_{\Gamma} \cup\{\perp\}$ where $D=\biguplus_{\sigma \in \mathcal{M}_{\Gamma}} D_{\sigma}^{\prime} \uplus D_{\perp}^{\prime}$, $D_{\sigma}^{\prime}=\left\{\langle\sigma, a\rangle \mid a \in D_{\sigma}\right\}$, and $D_{\perp}^{\prime}=\{\perp\}$. Furthermore, $D_{\sigma}^{\prime}$ has no infinite ascending chains on $\sqsubset$ if the indexed semiring is locally bounded. Thus, it is also possible to present our framework based on graded semirings. 


\section{Simplified Structure: Multiplication on Strictly Compatible Signatures}

An indexed semiring has a multiplication indexed by two stack signatures. However, it is often simpler to consider and implement a restricted multiplication defined only for strictly compatible signatures. We show that an indexed semiring over the ordered monoid of stack signatures can be constructed from such a structure.

We introduce weight structures that have a restricted multiplication $\odot_{\sigma_{1}, \sigma_{2}}$ for strictly compatible $\sigma_{1}$ and $\sigma_{2}$.

Definition 6.1. A weight structure $\mathcal{W}$ over a stack alphabet $\Gamma$ is $\left\langle\left\{D_{\sigma}\right\},\left\{\oplus_{\sigma}\right\},\left\{\odot_{\sigma_{1}, \sigma_{2}}\right\}\right.$, $\left.\left\{0_{\sigma}\right\},\left\{1_{\sigma}\right\},\left\{\uparrow_{\sigma, \sigma^{\prime}}\right\}\right\rangle$ such that

- $D_{\sigma}$ is a set for each proper stack signature $\sigma$.

- $\left\langle D_{\sigma}, \oplus_{\sigma}, 0_{\sigma}\right\rangle$ is a commutative monoid for each proper stack signature $\sigma$.

- $\odot_{\sigma_{1}, \sigma_{2}}$ is an associative binary operation of $D_{\sigma_{1}} \times D_{\sigma_{2}} \rightarrow D_{\sigma_{1} \sigma_{2}}$ for strictly compatible signatures $\sigma_{1}$ and $\sigma_{2}$.

- $1_{\sigma} \in D_{\sigma}$ is an indexed neutral element for $\epsilon / \epsilon \leq \sigma: a \odot_{\sigma^{\prime}, \sigma} 1_{\sigma}=a$ and $1_{\sigma} \odot_{\sigma, \sigma^{\prime \prime}} b=b$.

- $0_{\sigma}$ is an annihilator with respect to $\odot_{\sigma, \sigma^{\prime}}: 0_{\sigma_{1}} \odot_{\sigma_{1}, \sigma_{2}} a=0_{\sigma_{1} \sigma_{2}}=b \odot_{\sigma_{1}, \sigma_{2}} 0_{\sigma_{2}}$.

- $\odot$ distributes over $\oplus$.

$$
\begin{aligned}
& \left(a \oplus_{\sigma_{1}} b\right) \odot_{\sigma_{1}, \sigma_{2}} c=\left(a \odot_{\sigma_{1}, \sigma_{2}} c\right) \oplus_{\sigma_{1} \sigma_{2}}\left(b \odot_{\sigma_{1}, \sigma_{2}} c\right) \\
& a \odot_{\sigma_{1}, \sigma_{2}}\left(b \oplus_{\sigma_{2}} c\right)=\left(a \odot_{\sigma_{1}, \sigma_{2}} b\right) \oplus_{\sigma_{1} \sigma_{2}}\left(a \odot_{\sigma_{1}, \sigma_{2}} c\right)
\end{aligned}
$$

- $\uparrow_{\sigma, \sigma^{\prime}}$ is a conversion function of $D_{\sigma} \rightarrow D_{\sigma^{\prime}}$ for $\sigma \leq \sigma^{\prime}$ such that

$-\uparrow_{\sigma, \sigma}=$ id and $\uparrow_{\sigma, \sigma^{\prime \prime}}=\uparrow_{\sigma^{\prime}, \sigma^{\prime \prime}} \circ \uparrow_{\sigma, \sigma^{\prime}}$ for all $\sigma \leq \sigma^{\prime} \leq \sigma^{\prime \prime}$.

$-\uparrow_{\sigma, \sigma^{\prime}}\left(0_{\sigma}\right)=0_{\sigma^{\prime}}$ and $\uparrow_{\sigma, \sigma^{\prime}}(a \oplus b)=\uparrow_{\sigma, \sigma^{\prime}}(a) \oplus \uparrow_{\sigma, \sigma^{\prime}}(b)$

$-\uparrow_{\sigma_{1} \cdot \sigma_{2}, \sigma_{1}^{\prime} \cdot \sigma_{2}^{\prime}}(a \odot b)=\uparrow_{\sigma_{1}, \sigma_{1}^{\prime}}(a) \odot \uparrow_{\sigma_{2}, \sigma_{2}^{\prime}}(b)$ for $\sigma_{1} \leq \sigma_{1}^{\prime}, \sigma_{2} \leq \sigma_{2}^{\prime}, \sigma_{1}$ and $\sigma_{2}$ are strictly compatible, and $\sigma_{1}^{\prime}$ and $\sigma_{2}^{\prime}$ are strictly compatible.

$-\uparrow_{\sigma, \sigma^{\prime}}\left(1_{\sigma}\right)=1_{\sigma^{\prime}}$ for $\epsilon / \epsilon \leq \sigma \leq \sigma^{\prime}$.

We show that the multiplication of an indexed semiring over $\mathcal{M}_{\Gamma}$ can be obtained from that of a weight structure. Let $\left\{D_{\sigma}^{\prime}\right\}$ be a family of $\left\{D_{\sigma}\right\} \cup\left\{D_{\top}\right\}$ where $D_{\top}=\{\bullet\}$. Then, the multiplication on $D_{\sigma}^{\prime}$ is defined as follows.

$$
x \otimes_{\sigma_{1}, \sigma_{2}} y= \begin{cases}\uparrow \sigma_{1}, \sigma_{1}^{\prime}(x) \odot_{\sigma_{1}^{\prime}, \sigma_{2}} y & \text { if } \sigma_{1} \leq \sigma_{1}^{\prime} \text { and } \sigma_{1}^{\prime} \| \sigma_{2} \\ x \odot_{\sigma_{1}, \sigma_{2}^{\prime}} \uparrow_{\sigma_{2}, \sigma_{2}^{\prime}}(y) & \text { if } \sigma_{2} \leq \sigma_{2}^{\prime} \text { and } \sigma_{1} \| \sigma_{2}^{\prime} \\ \bullet & \text { otherwise }\end{cases}
$$

The other operations are extended for $D_{\top}$ in a straightforward manner. Then, we obtain a semiring indexed by the ordered monoid $\mathcal{M}_{\Gamma}$.

Theorem 6.2. Let $\left\langle\left\{D_{\sigma}\right\},\left\{\oplus_{\sigma}\right\},\left\{\odot_{\sigma_{1}, \sigma_{2}}\right\},\left\{0_{\sigma}\right\},\left\{1_{\sigma}\right\},\left\{\uparrow_{\sigma, \sigma^{\prime}}\right\}\right\rangle$ be a weight structure. Then, $\left\langle\left\{D_{\sigma}^{\prime}\right\},\left\{\oplus_{\sigma}\right\},\left\{\otimes_{\sigma_{1}, \sigma_{2}}\right\},\left\{0_{\sigma}\right\}, 1_{\epsilon / \epsilon},\left\{\uparrow_{\sigma, \sigma^{\prime}}\right\}\right\rangle$ is an indexed semiring over an ordered monoid $\mathcal{M}_{\Gamma}$.

Two key properties of the indexed semiring are proved by the following lemmas. The other properties are easily proved from the corresponding properties of a weight structure.

Lemma 6.3. $\left(a \otimes_{\sigma_{1}, \sigma_{2}} b\right) \otimes_{\sigma_{1} \sigma_{2}, \sigma_{3}} c=a \otimes_{\sigma_{1}, \sigma_{2} \sigma_{3}}\left(b \otimes_{\sigma_{2}, \sigma_{3}} c\right)$.

Proof. We prove the claim by analyzing the cases where $\sigma_{1} \sigma_{2} \sigma_{3} \neq \top$ by Lemma A.2. The proofs of two cases are omitted because they are symmetric to other cases. 
Case: $\sigma_{1} \leq \sigma_{1}^{\prime}, \sigma_{3} \leq \sigma_{3}^{\prime}, \sigma_{1}^{\prime} \| \sigma_{2}$, and $\sigma_{2} \| \sigma_{3}^{\prime}$.

$$
\begin{aligned}
\left(a \otimes_{\sigma_{1}, \sigma_{2}} b\right) \otimes_{\sigma_{1} \sigma_{2}, \sigma_{3}} c & =\left(\uparrow_{\sigma_{1}, \sigma_{1}^{\prime}}(a) \odot_{\sigma_{1}^{\prime}, \sigma_{2}} b\right) \otimes_{\sigma_{1} \sigma_{2}, \sigma_{3}} c \\
& =\left(\uparrow_{\sigma_{1}, \sigma_{1}^{\prime}}(a) \odot_{\sigma_{1}^{\prime}, \sigma_{2}} b\right) \odot_{\sigma_{1}^{\prime} \sigma_{2}, \sigma_{3}^{\prime}} \uparrow_{\sigma_{3}, \sigma_{3}^{\prime}}(c) \\
& =\uparrow_{\sigma_{1}, \sigma_{1}^{\prime}}(a) \odot_{\sigma_{1}^{\prime}, \sigma_{2} \sigma_{3}^{\prime}}\left(b \odot_{\sigma_{2}, \sigma_{3}^{\prime}} \uparrow_{\sigma_{3}, \sigma_{3}^{\prime}}(c)\right) \\
& =a \otimes_{\sigma_{1}, \sigma_{2} \sigma_{3}}\left(b \otimes_{\sigma_{2}, \sigma_{3}} c\right)
\end{aligned}
$$

Case: $\sigma_{1} \leq \sigma_{1}^{\prime}, \sigma_{2} \leq \sigma_{2}^{\prime}, \sigma_{1}^{\prime} \| \sigma_{2}$, and $\sigma_{2}^{\prime} \| \sigma_{3}$. We have $\sigma_{1}^{\prime} \leq \sigma_{1}^{\prime \prime}$ and $\sigma_{1}^{\prime \prime} \| \sigma_{2}^{\prime}$ for some $\sigma_{1}^{\prime \prime}$.

$$
\begin{aligned}
\left(a \otimes_{\sigma_{1}, \sigma_{2}} b\right) \otimes_{\sigma_{1} \sigma_{2}, \sigma_{3}} c & =\left(\uparrow_{\sigma_{1}, \sigma_{1}^{\prime}}(a) \odot_{\sigma_{1}^{\prime}, \sigma_{2}} b\right) \otimes_{\sigma_{1} \sigma_{2}, \sigma_{3}} c \\
& =\uparrow_{\sigma_{1}^{\prime} \sigma_{2}, \sigma_{1}^{\prime \prime} \sigma_{2}^{\prime}}\left(\uparrow_{\sigma_{1}, \sigma_{1}^{\prime}}(a) \odot_{\sigma_{1}^{\prime}, \sigma_{2}} b\right) \odot_{\sigma_{1}^{\prime \prime} \sigma_{2}^{\prime}, \sigma_{3}} c \\
& =\left(\uparrow_{\sigma_{1}, \sigma_{1}^{\prime \prime}}(a) \odot_{\sigma_{1}^{\prime \prime}, \sigma_{2}^{\prime}} \uparrow_{\sigma_{2}, \sigma_{2}^{\prime}}(b)\right) \odot_{\sigma_{1}^{\prime \prime} \sigma_{2}^{\prime}, \sigma_{3}} c \\
& =\uparrow_{\sigma_{1}, \sigma_{1}^{\prime \prime}}(a) \odot_{\sigma_{1}^{\prime \prime}, \sigma_{2}^{\prime} \sigma_{3}}\left(\uparrow_{\sigma_{2}, \sigma_{2}^{\prime}}(b) \odot_{\sigma_{2}^{\prime}, \sigma_{3}} c\right) \\
& =a \otimes_{\sigma_{1}, \sigma_{2} \sigma_{3}}\left(b \otimes_{\sigma_{2}, \sigma_{3}} c\right)
\end{aligned}
$$

Case: $\sigma_{2} \leq \sigma_{2}^{\prime} \leq \sigma_{2}^{\prime \prime}, \sigma_{1} \| \sigma_{2}^{\prime}$, and $\sigma_{2}^{\prime \prime} \| \sigma_{3}$. We have $\sigma_{1} \leq \sigma_{1}^{\prime \prime}$ and $\sigma_{1}^{\prime \prime} \| \sigma_{2}^{\prime \prime}$ for some $\sigma_{1}^{\prime \prime}$.

$$
\begin{aligned}
\left(a \otimes_{\sigma_{1}, \sigma_{2}} b\right) \otimes_{\sigma_{1} \sigma_{2}, \sigma_{3}} c & =\left(a \odot_{\sigma_{1}, \sigma_{2}^{\prime}} \uparrow_{\sigma_{2}, \sigma_{2}^{\prime}}(b)\right) \otimes_{\sigma_{1} \sigma_{2}, \sigma_{3}} c \\
& =\uparrow_{\sigma_{1} \sigma_{2}^{\prime}, \sigma_{1}^{\prime \prime} \sigma_{2}^{\prime \prime}}\left(a \odot_{\sigma_{1}^{\prime}, \sigma_{2}} \uparrow_{\sigma_{2}, \sigma_{2}^{\prime}}(b)\right) \odot_{\sigma_{1}^{\prime \prime} \sigma_{2}^{\prime \prime}, \sigma_{3}} c \\
& =\left(\uparrow_{\sigma_{1}, \sigma_{1}^{\prime \prime}}(a) \odot_{\sigma_{1}^{\prime \prime}, \sigma_{2}^{\prime \prime}} \uparrow_{\sigma_{2}, \sigma_{2}^{\prime \prime}}(b)\right) \odot_{\sigma_{1}^{\prime \prime} \sigma_{2}^{\prime \prime}, \sigma_{3}} c \\
& =\uparrow_{\sigma_{1}, \sigma_{1}^{\prime \prime}}(a) \odot_{\sigma_{1}^{\prime \prime}, \sigma_{2}^{\prime \prime} \sigma_{3}}\left(\uparrow_{\sigma_{2}, \sigma_{2}^{\prime \prime}}(b) \odot_{\sigma_{2}^{\prime \prime}, \sigma_{3}} c\right) \\
& =a \otimes_{\sigma_{1}, \sigma_{2} \sigma_{3}}\left(b \otimes_{\sigma_{2}, \sigma_{3}} c\right)
\end{aligned}
$$

Lemma 6.4. If $\sigma_{1} \leq \sigma_{1}^{\prime}$ and $\sigma_{1}^{\prime} \cdot \sigma_{2} \neq \top$, then $\uparrow_{\sigma_{1} \sigma_{2}, \sigma_{1}^{\prime} \sigma_{2}}\left(x \otimes_{\sigma_{1}, \sigma_{2}} y\right)=\uparrow_{\sigma_{1}, \sigma_{1}^{\prime}}(x) \otimes_{\sigma^{\prime}, \sigma_{2}} y$.

Proof.

Case: $\sigma_{1} \leq \sigma_{1}^{\prime \prime}$ and $\sigma_{1}^{\prime \prime} \| \sigma_{2}$. We have $\left(\sigma_{1}^{\prime} \sqcup \sigma_{1}^{\prime \prime}\right) \cdot \sigma_{2}=\sigma_{1}^{\prime} \cdot \sigma_{2} \sqcup \sigma_{1}^{\prime \prime} \cdot \sigma_{2}=\sigma_{1}^{\prime} \cdot \sigma_{2} \sqcup \sigma_{1} \cdot \sigma_{2}=$ $\left(\sigma_{1}^{\prime} \sqcup \sigma_{1}\right) \cdot \sigma_{2}=\sigma_{1}^{\prime} \cdot \sigma_{2}$, Then, either $\sigma_{1}^{\prime} \leq \sigma_{1}^{\prime \prime}$ or $\sigma_{1}^{\prime \prime} \leq \sigma_{1}^{\prime}$ holds.

Subcase: $\sigma_{1}^{\prime} \leq \sigma_{1}^{\prime \prime}$. We have $\sigma_{1} \cdot \sigma_{2}=\sigma_{1}^{\prime} \cdot \sigma_{2}=\sigma_{1}^{\prime \prime} \cdot \sigma_{2}$.

$$
\begin{aligned}
\uparrow_{\sigma_{1} \sigma_{2}, \sigma_{1}^{\prime} \sigma_{2}}\left(x \otimes_{\sigma_{1}, \sigma_{2}} y\right) & =\uparrow_{\sigma_{1}^{\prime \prime} \sigma_{2}, \sigma_{1}^{\prime} \sigma_{2}}\left(\uparrow_{\sigma_{1}, \sigma_{1}^{\prime \prime}}(x) \odot_{\sigma_{1}^{\prime \prime}, \sigma_{2}} y\right) \\
& =\uparrow_{\sigma_{1}, \sigma_{1}^{\prime \prime}}(x) \odot_{\sigma_{1}^{\prime \prime}, \sigma_{2}} y \\
& =\uparrow_{\sigma_{1}^{\prime}, \sigma_{1}^{\prime \prime}}\left(\uparrow_{\sigma_{1}, \sigma_{1}^{\prime}}(x)\right) \odot_{\sigma_{1}^{\prime \prime}, \sigma_{2}} y \\
& =\uparrow_{\sigma_{1}, \sigma_{1}^{\prime}}(x) \otimes_{\sigma_{1}^{\prime}, \sigma_{2}} y
\end{aligned}
$$

Subcase: $\sigma_{1}^{\prime \prime} \leq \sigma_{1}^{\prime}$. From $\sigma_{1}^{\prime \prime} \| \sigma_{2}$ and $\sigma_{1}^{\prime \prime} \leq \sigma_{1}^{\prime}, \sigma_{2} \leq \sigma_{2}^{\prime}$ and $\sigma_{1}^{\prime} \| \sigma_{2}^{\prime}$ for some $\sigma_{2}^{\prime}$.

$$
\begin{aligned}
\uparrow_{\sigma_{1} \sigma_{2}, \sigma_{1}^{\prime} \sigma_{2}}\left(x \otimes_{\sigma_{1}, \sigma_{2}} y\right) & =\uparrow_{\sigma_{1}^{\prime \prime} \sigma_{2}, \sigma_{1}^{\prime} \sigma_{2}}\left(\uparrow_{\sigma_{1}, \sigma_{1}^{\prime \prime}}(x) \odot_{\sigma_{1}^{\prime \prime}, \sigma_{2}} y\right) \\
& =\uparrow_{\sigma_{1}^{\prime \prime} \sigma_{2}, \sigma_{1}^{\prime} \sigma_{2}^{\prime}}\left(\uparrow_{\sigma_{1}, \sigma_{1}^{\prime \prime}}(x) \odot_{\sigma_{1}^{\prime \prime}, \sigma_{2}} y\right) \\
& =\uparrow_{\sigma_{1}, \sigma_{1}^{\prime}}(x) \odot_{\sigma_{1}^{\prime}, \sigma_{2}^{\prime}} \uparrow_{\sigma_{2}, \sigma_{2}^{\prime}}(y) \\
& =\uparrow_{\sigma_{1}, \sigma_{1}^{\prime}}(x) \otimes_{\sigma_{1}^{\prime}, \sigma_{2}} y
\end{aligned}
$$

Case: $\sigma_{2} \leq \sigma_{2}^{\prime}$ and $\sigma_{1} \| \sigma_{2}^{\prime}$. From $\sigma_{1} \| \sigma_{2}^{\prime}$ and $\sigma_{1} \leq \sigma_{1}^{\prime}, \sigma_{2}^{\prime} \leq \sigma_{2}^{\prime \prime}$ and $\sigma_{1}^{\prime} \| \sigma_{2}^{\prime \prime}$ for some $\sigma_{2}^{\prime \prime}$.

$$
\begin{aligned}
\uparrow_{\sigma_{1} \sigma_{2}, \sigma_{1}^{\prime} \sigma_{2}}\left(x \otimes_{\sigma_{1}, \sigma_{2}} y\right) & =\uparrow_{\sigma_{1} \sigma_{2}^{\prime}, \sigma_{1}^{\prime} \sigma_{2}^{\prime \prime}}\left(x \odot_{\sigma_{1}, \sigma_{2}^{\prime}} \uparrow_{\sigma_{2}, \sigma_{2}^{\prime}}(y)\right) \\
& =\uparrow_{\sigma_{1}, \sigma_{1}^{\prime}}(x) \odot_{\sigma_{1}^{\prime}, \sigma_{2}^{\prime \prime}} \uparrow_{\sigma_{2}, \sigma_{2}^{\prime \prime}}(y) \\
& =\uparrow_{\sigma_{1}, \sigma_{1}^{\prime}}(x) \otimes_{\sigma_{1}^{\prime}, \sigma_{2}} y
\end{aligned}
$$


We present a weight structure for the indexed semiring in Example 5.3. It is almost trivial to check that it really forms a weight structure. On the other hand, if we directly define the indexed semiring, we have to repeat proofs similar to those of Lemma 6.3 and 6.4

Example 6.5. $\left\langle\left\{D_{\sigma}\right\},\left\{\oplus_{\sigma}\right\},\left\{\odot_{\sigma_{1}, \sigma_{2}}\right\},\left\{0_{\sigma}\right\},\left\{1_{\sigma}\right\},\left\{\uparrow_{\sigma, \sigma^{\prime}}\right\}\right\rangle$ given by the following components forms a weight structure.

- $D_{w / w^{\prime}}=\mathbb{N} \geq \max \left(|w|,\left|w^{\prime}\right|\right) \cup\{\infty\}$.

- $a \oplus_{\sigma} b=\min (a, b)$ and $0_{\sigma}=\infty .\left\langle D_{\sigma}, \oplus_{\sigma}, 0_{\sigma}\right\rangle$ is clearly a commutative monoid.

- $a \odot_{\sigma_{1}, \sigma_{2}} b=\max (a, b)$. It is clearly associative and its anihilator is $\infty$.

- $1_{w / w}=|w| .1_{w / w} \odot_{w / w, w / w^{\prime}} b=\max (|w|, b)=b$ since $b \in \mathbb{N} \geq \max \left(|w|,\left|w^{\prime}\right|\right)$.

- $\uparrow_{w_{1} / w_{2}, w_{1} w / w_{2} w}(a)=a+|w|$. We only show $\uparrow_{\sigma_{1} \cdot \sigma_{2}, \sigma_{1}^{\prime} \cdot \sigma_{2}^{\prime}}(a \odot b)=\uparrow_{\sigma_{1}, \sigma_{1}^{\prime}}(a) \odot \uparrow_{\sigma_{2}, \sigma_{2}^{\prime}}(b)$. Let $\sigma_{1}=w_{1} / w$ and $\sigma_{2}=w / w_{2}$. Then, $\sigma_{1}^{\prime}=w_{1} w^{\prime} / w w^{\prime}$ and $\sigma_{2}^{\prime}=w w^{\prime} / w_{2} w^{\prime}$ for some $w^{\prime}$.

$$
\begin{aligned}
\uparrow_{\sigma_{1} \cdot \sigma_{2}, \sigma_{1}^{\prime} \cdot \sigma_{2}^{\prime}}(a \odot b) & =\max (a, b)+\left|w^{\prime}\right| \\
& =\max \left(a+\left|w^{\prime}\right|, b+\left|w^{\prime}\right|\right) \\
& =\uparrow_{\sigma_{1}, \sigma_{1}^{\prime}}(a) \odot \uparrow_{\sigma_{2}, \sigma_{2}^{\prime}}(b)
\end{aligned}
$$

\section{Applications}

We present four applications of the readability analysis of weighted pushdown automata over indexed semirings. The indexed semirings used in these examples are locally bounded and thus our framework guarantees termination of the analyses.

7.1. Encoding of Local Variables into Weight. Suwimonteerabuth applied a semiring similar to one constructed from an indexed semiring to encode local variables of a recursive program into weight [Suw09]. Although his implementation worked without any problem, it is actually not in the standard framework of weighted pushdown systems because the semiring is not bounded.

We show that his encoding can be formulated more naturally with an indexed semiring. In order to simplify our presentation, we give an encoding of a pushdown system into a weighted pushdown system with a singleton stack alphabet. Since local variables can be encoded into a stack alphabet, the same approach can be applied for the encoding of local variables.

Let us consider a singleton stack alphabet $\Gamma^{\prime}=\{\#\}$. We write $m / n$ for a stack signature $\#^{m} / \#^{n}$. We will construct a weight structure to translate pushdown systems over a stack alphabet $\Gamma$. We define a weight structure $\mathcal{W}_{\Gamma}=\left\langle\left\{D_{\sigma}\right\},\left\{\oplus_{\sigma}\right\},\left\{\odot_{\sigma_{1}, \sigma_{2}}\right\},\left\{0_{\sigma}\right\},\left\{1_{\sigma}\right\}\right.$, $\left.\left\{\uparrow \sigma_{1}, \sigma_{2}\right\}\right\rangle$ as follows.

- $D_{m / n}$ is the set of relations between $\Gamma^{m}$ and $\Gamma^{n}: D_{m / n}=2^{\Gamma^{m} \times \Gamma^{n}}$.

- $0_{m / n}=\varnothing$ and $1_{m / m}=\left\{\langle x, x\rangle \mid x \in \Gamma^{m}\right\}$.

- $R_{1} \odot_{l / m, m / n} R_{2}$ is a composition of two relations $R_{1}$ and $R_{2}: R_{1} \circ R_{2}$ where $R_{1} \subseteq \Gamma^{l} \times \Gamma^{m}$ and $R_{2} \subseteq \Gamma^{m} \times \Gamma^{n}$.

- $R_{1} \oplus_{m / n} R_{2}$ is the union of two relations $R_{1}$ and $R_{2}: R_{1} \cup R_{2}$ where $R_{1}, R_{2} \subseteq \Gamma^{m} \times \Gamma^{n}$.

- $\uparrow_{l / m, l+1 / m+1}$ extends the domain of a relation and is defined by

$$
\uparrow_{l / m, l+1 / m+1}(R)=\{\langle\langle x, z\rangle,\langle y, z\rangle\rangle \mid\langle x, y\rangle \in R \wedge z \in \Gamma\}
$$

where we consider $\Gamma^{k+1}=\Gamma^{k} \times \Gamma$. 
It is straightforward to show this structure forms a weight structure. Furthermore, it induces a locally bounded indexed semiring because $D_{m / n}$ is the power set of a finite set and ordered by the set inclusion.

We show how to simulate a pushdown system $\mathcal{P}=\langle P, \Gamma, \Delta\rangle$ by a weighted pushdown system $\mathcal{P}^{\prime}$ over the weight structure $\mathcal{W}_{\Gamma}$. Let $\mathcal{P}^{\prime}$ be $\left\langle P, \Gamma^{\prime}, \Delta^{\prime}\right\rangle$ such that

$$
\left\langle p, \#, p^{\prime}, \#^{m}, a\right\rangle \in \Delta^{\prime} \quad \text { iff } \quad\left\langle p, \gamma, p^{\prime}, w\right\rangle \in \Delta
$$

where $|w|=m$ and $a=\{\langle\gamma, w\rangle\}$.

Then, $\mathcal{P}$ and $\mathcal{P}^{\prime}$ are equivalent in the following sense:

$$
p \stackrel{w / w^{\prime}}{\underset{\mathcal{P}}{\rightleftarrows}} p^{\prime} \quad \Longleftrightarrow \quad p \stackrel{m / m^{\prime} \mid a}{\underset{\mathcal{P}^{\prime}}{\longrightarrow}} p^{\prime} \wedge\left\langle w, w^{\prime}\right\rangle \in a
$$

where $m=|w|$ and $m^{\prime}=\left|w^{\prime}\right|$. Then, we can check the reachability in $\mathcal{P}$ by checking that in $\mathcal{P}^{\prime}$.

7.2. Conditional Pushdown Systems. Esparza et al. introduced pushdown systems with checkpoints that have the ability to inspect the whole stack content against a regular language [EKS03. Li and Ogawa reformulated their definition and called them conditional pushdown systems [LO10]. We review conditional pushdown systems and then formulate the reachability analysis in our previous work [MM12] as that of weighted pushdown systems.

Definition 7.1. A conditional pushdown system $\mathcal{P}$ is a structure $\langle P, \Gamma, \Delta\rangle$ where $P$ is a finite set of states, $\Gamma$ is a stack alphabet, and $\Delta \subseteq P \times \Gamma \times P \times \Gamma^{*} \times \operatorname{Reg}(\Gamma)$ is a set of transitions where $\operatorname{Reg}(\Gamma)$ is the set of regular languages over $\Gamma$.

We write $\langle p, \gamma\rangle \stackrel{R}{\hookrightarrow}\left\langle p^{\prime}, w\right\rangle$ if $\left\langle p, \gamma, p^{\prime}, w, R\right\rangle \in \Delta$ as weighted pushdown systems. The transition relation of a conditional pushdown system is defined as follows.

- $\langle p, w\rangle \Rightarrow\langle p, w\rangle$.

- $\left\langle p, \gamma w^{\prime}\right\rangle \Rightarrow\left\langle p^{\prime}, w w^{\prime}\right\rangle$ if $\langle p, \gamma\rangle \stackrel{R}{\hookrightarrow}\left\langle p^{\prime}, w\right\rangle$ and $w^{\prime} \in R$.

- $\langle p, w\rangle \Rightarrow\left\langle p^{\prime}, w^{\prime}\right\rangle$ if $\langle p, w\rangle \Rightarrow\left\langle p^{\prime \prime}, w^{\prime \prime}\right\rangle$ and $\left\langle p^{\prime \prime}, w^{\prime \prime}\right\rangle \Rightarrow\left\langle p^{\prime}, w^{\prime}\right\rangle$.

In the second case above, the transition can be taken only when the current stack content excluding its top is included in the regular language $R$ given as the condition of the rule.

We show that the transition of a conditional pushdown system can be simulated by that of a weighted pushdown system without conditional rules. Let us design a weight structure for this simulation. We use the same domain for all proper stack signatures $\sigma: D_{\sigma}=2^{\Gamma^{*}}$. Then, the weight structure $\left\langle\left\{D_{\sigma}\right\},\left\{\oplus_{\sigma}\right\},\left\{\odot_{\sigma_{1}, \sigma_{2}}\right\},\left\{0_{\sigma}\right\},\left\{1_{\sigma}\right\},\left\{\uparrow_{\sigma, \sigma^{\prime}}\right\}\right\rangle$ is given as follows.

- $0_{\sigma}=\varnothing$ and $1_{\sigma}=\Gamma^{*}$.

- $a \oplus_{\sigma} b=a \cup b$.

- $a \odot_{\sigma_{1}, \sigma_{2}} b=a \cap b$ for strictly compatible signatures $\sigma_{1}$ and $\sigma_{2}$.

- $\uparrow_{w_{1} / w_{2}, w_{1} w / w_{2} w}(a)=w^{-1} a$ where $w^{-1} a$ is left quotient defined by $w^{-1} a=\left\{w^{\prime} \mid w w^{\prime} \in a\right\}$. From basic properties of left quotient and set operations, it is clear that this structure forms a weight structure. Then, for a conditional pushdown system $\mathcal{P}$ we obtain a weighted pushdown system $\mathcal{P}^{\prime}$ over the indexed semiring above by considering a conditional transition rule $\langle p, \gamma\rangle \stackrel{R}{\leftrightarrow}\left\langle p^{\prime}, w\right\rangle$ as a weighted one.

A conditional pushdown system $\mathcal{P}$ is simulated by a weighted pushdown system $\mathcal{P}^{\prime}$ in the following sense. 
- If $\left\langle p_{1}, w_{1}\right\rangle \underset{\mathcal{P}}{\Rightarrow}\left\langle p_{2}, w_{2}\right\rangle$, then there exist $w, w_{1}^{\prime}$, and $w_{2}^{\prime}$ such that $p_{1} \stackrel{w_{1}^{\prime} / w_{2}^{\prime}}{\overrightarrow{\mathcal{P}^{\prime}}} \mid a p_{2}, w \in a$, and $w_{1} / w_{2}=w_{1}^{\prime} w / w_{2}^{\prime} w$.

- If $p_{1} \underset{\mathcal{P}^{\prime}}{\stackrel{w_{1} / w_{2}}{\rightleftharpoons}} \mid a p_{2}$ and $w \in a$, then $\left\langle p_{1}, w_{1} w\right\rangle \underset{\mathcal{P}}{\Rightarrow}\left\langle p_{2}, w_{2} w\right\rangle$.

Please note that this weight structure is not locally bounded because $2^{\Gamma^{*}}$ is not bounded with respect to the set inclusion. However, $D_{\sigma}$ can be restricted to the set $D \subseteq 2^{\Gamma^{*}}$ inductively defined as follows.

- $\varnothing \in D$ and $\Gamma^{*} \in D$.

- $R \in D$ if $\langle p, \gamma\rangle \stackrel{R}{\hookrightarrow}\left\langle p^{\prime}, w\right\rangle$ for some $p, \gamma, p^{\prime}, w$.

- $R_{1} \cap R_{2} \in D$ and $R_{1} \cup R_{2} \in D$ if $R_{1} \in D$ and $R_{2} \in D$.

- $w^{-1} R \in D$ if $R \in D$ and $w \in \Gamma^{*}$.

This set $D$ is finite because the set of transitions is finite, there are finitely many languages obtained from each regular language with left quotient, and left quotient distributes over union and intersection. Thus, we obtain a locally bounded indexed semiring by using $D$. This gives the algorithm of the backward reachability analysis for conditional pushdown systems that we used to analyze the HTML5 parser specification [MM12].

7.3. Well-Structured Pushdown Systems. Cai and Ogawa introduced well-structured pushdown systems (WSPDS) where the set of states and stack alphabet can be possibly infinite well-quasi-ordered sets. They showed that the coverability problem is decidable for WSPDS with a finite set of states and then extended the result for several subclasses of WSPDS CO13. We show that the coverability of WSPDS with a finite set of states can also be decided through a translation to weighted pushdown systems with indexed weight domains.

A quasi-ordering $(D, \preceq)$ is a reflexive and transitive binary relation on $D$. A quasi-order $(D, \preceq)$ is a well-quasi-order if, for each infinite sequence $a_{1}, a_{2}, a_{3}, \ldots$ in $D$, there exist $i, j$ such that $i<j$ and $a_{i} \preceq a_{j}$. A set $I \subseteq D$ is an ideal if $a \in I$ and $a \preceq b$ imply $b \in I$. The upward closure of $A \subseteq D$ is $A^{\uparrow}=\{b \in D \mid \exists a \in A . a \preceq b\}$. The family of ideals over $A$ is denoted by $\mathcal{I}(A)$.

Well-structured pushdown systems are defined as follows where $\operatorname{PFun}(A, B)$ denotes the set of partial functions from $A$ to $B$.

Definition 7.2. A well-structured pushdown system is a structure $\langle P, \Gamma, \Delta\rangle$ where $P$ is a finite set of states, $\Gamma$ is a possibly infinite set of stack symbols with well-quasi-order $\preceq$, and $\Delta \subseteq P \times P \times \bigcup_{i \in \mathbb{N}} \operatorname{PFun}\left(\Gamma, \Gamma^{i}\right)$ is a finite set of monotonic transition rules. A transition rule $\left\langle p, p^{\prime}, \phi\right\rangle$ is monotonic if $\phi$ is monotonic on $\preceq$.

If $\left\langle p, p^{\prime}, \phi\right\rangle \in \Delta$ and $\phi \in \operatorname{PFun}\left(\Gamma, \Gamma^{i}\right)$, then $\phi^{-1}(X) \in \mathcal{I}(\Gamma)$ for any $X \in \mathcal{I}\left(\Gamma^{i}\right)$ by the monotonicity of $\phi$. The transition relation of a WSPDS is defined as follows.

- $\langle p, w\rangle \Rightarrow\langle p, w\rangle$.

- $\left\langle p, \gamma w^{\prime}\right\rangle \Rightarrow\left\langle p^{\prime}, \phi(\gamma) w^{\prime}\right\rangle$ if $\left\langle p, p^{\prime}, \phi\right\rangle \in \Delta$ and $\phi(\gamma)$ is defined.

- $\langle p, w\rangle \Rightarrow\left\langle p^{\prime}, w^{\prime}\right\rangle$ if $\langle p, w\rangle \Rightarrow\left\langle p^{\prime \prime}, w^{\prime \prime}\right\rangle$ and $\left\langle p^{\prime \prime}, w^{\prime \prime}\right\rangle \Rightarrow\left\langle p^{\prime}, w^{\prime}\right\rangle$.

Cai and Ogawa showed that the coverability problem of WSPDS is decidable. We say that $\left\langle p_{2}, w_{2}\right\rangle$ is covered by $\left\langle p_{1}, w_{1}\right\rangle$ if we have $\left\langle p_{1}, w_{1}\right\rangle \Rightarrow\left\langle p_{2}, w_{2}^{\prime}\right\rangle$ for some $w_{2}^{\prime}$ such that $w_{2} \preceq w_{2}^{\prime}$. The key to the development of the coverability analysis of WSPDS by Cai and 
Ogawa is the following lemma. This also makes it possible to construct a locally bounded indexed semiring.

Lemma 7.3 (Finkel et al. [FS01]). If $\preceq$ is a well-quasi-order, then any infinite sequence $I_{0} \subseteq I_{1} \subseteq I_{2} \subseteq \cdots$ of ideals eventually stabilizes.

For the coverability analysis, we translate a WSPDS into a weighted pushdown system with a singleton stack alphabet $\Gamma^{\prime}=\{\#\}$. Then we translate the transition rule $\left\langle p, p^{\prime}, \phi\right\rangle \in \Delta$ in WSPDS into the following transition in a weighted pushdown system $\mathcal{P}^{\prime}$ :

$$
\langle p, \#\rangle \stackrel{\phi^{-1}}{\underset{\mathcal{P}^{\prime}}{\longrightarrow}}\left\langle p^{\prime}, \#^{i}\right\rangle
$$

where $\phi \in \operatorname{PFun}\left(\Gamma, \Gamma^{i}\right)$. We adopt $\phi^{-1}$ as a weight instead of $\phi$ because we apply $\phi^{-1}(X) \in$ $\mathcal{I}(\Gamma)$ for any $X \in \mathcal{I}\left(\Gamma^{i}\right)$. The weight structure $\left\langle\left\{D_{\sigma}\right\},\left\{\oplus_{\sigma}\right\},\left\{\odot_{\sigma_{1}, \sigma_{2}}\right\},\left\{0_{\sigma}\right\},\left\{1_{\sigma}\right\},\left\{\uparrow_{\sigma_{1}, \sigma_{2}}\right\}\right\rangle$ is defined as follows.

- $D_{m / n}=\Gamma^{n} \rightarrow \mathcal{I}\left(\Gamma^{m}\right)$.

- $0_{m / n}=\lambda x \cdot \varnothing$ and $1_{m / m}=\lambda x \cdot\{x\}^{\uparrow}$.

- $f_{1} \odot_{l / m, m / n} f_{2}$ is the composition of functions: $\hat{f}_{1} \circ f_{2}$ where $\hat{f}_{1}(X)=\bigcup_{x \in X} f_{1}(x)$.

- $f_{1} \oplus_{m / n} f_{2}$ is defined by $\lambda x . f_{1}(x) \cup f_{2}(x)$.

- $\uparrow_{l / m, l+1 / m+1}$ extends the domain and range of a function and is defined as follows:

$$
\uparrow_{l / m, l+1 / m+1}(f)=\lambda\langle y, z\rangle \cdot f(y) \times\{z\}^{\uparrow}
$$

where $y \in \Gamma^{m}$ and $z \in \Gamma$.

$\left\langle D_{m / n}, \oplus_{m / n}, 0_{m / n}\right\rangle$ is clearly a commutative monoid. The other properties of a weight structure can be easily verified. Furthermore, it induces a locally bounded indexed semiring because $D_{m / 0}$ is isomorphic to $\mathcal{I}\left(\Gamma^{m}\right)$ and there are no infinite ascending chains of ideals by Lemma 7.3. It should be noted that $D_{m / n}$ is not bounded in general for $n>0$.

We translate a WSPDS $\mathcal{P}=\langle P, \Gamma, \Delta\rangle$ to a weighted pushdown system $\mathcal{P}^{\prime}=\left\langle P, \Gamma^{\prime}, \Delta^{\prime}\right\rangle$ over the above weight structure. The set of transition rules $\Delta^{\prime}$ is defined by

$$
\left\langle p, \#, p^{\prime}, \#^{i}, a\right\rangle \in \Delta^{\prime} \quad \text { if } \quad\left\langle p, p^{\prime}, \phi\right\rangle \in \Delta \text { and } \phi \in \operatorname{PFun}\left(\Gamma, \Gamma^{i}\right)
$$

where $a=\lambda w \cdot \phi^{-1}\left(\{w\}^{\uparrow}\right)$.

Then, $\mathcal{P}$ and $\mathcal{P}^{\prime}$ are closely related in the following sense. The proof appears in Appendix B.

\section{Proposition 7.4.}

- If $\left\langle p_{1}, w_{1}\right\rangle \underset{\mathcal{P}}{\Rightarrow}\left\langle p_{2}, w_{2}\right\rangle$, then $\left\langle p_{1}, m_{1}\right\rangle \underset{\mathcal{P}^{\prime}}{\stackrel{a}{\Rightarrow}}\left\langle p_{2}, m_{2}\right\rangle$ and $w_{1} \in a\left(w_{2}\right)$.

- If $\left\langle p_{1}, m_{1}\right\rangle \underset{\mathcal{P}^{\prime}}{\stackrel{a}{\Rightarrow}}\left\langle p_{2}, m_{2}\right\rangle$ and $w_{1} \in a\left(w_{2}\right)$, then $\left\langle p_{1}, w_{1}\right\rangle \underset{\mathcal{P}}{\Rightarrow}\left\langle p_{2}, w_{2}^{\prime}\right\rangle$ for some $w_{2} \preceq w_{2}^{\prime}$.

where $m_{1}=\left|w_{1}\right|$ and $m_{2}=\left|w_{2}\right|$.

Then, the coverability in $\mathcal{P}$ can be checked by applying the reachability analysis to $\mathcal{P}^{\prime}$ in the following manner. Let us consider the coverability of $\langle p, w\rangle$ for $w=\gamma_{1} \gamma_{2} \cdots \gamma_{n}$. We represent $w$ by a weighted automaton $\mathcal{A}_{w}=\left\langle\left\{q_{0}, q_{1}, \ldots, q_{n}\right\},\{\#\}, \Delta_{w}, q_{0},\left\{q_{n}\right\}\right\rangle$ where $\left\langle q_{i-1}, q_{i}, \#,\left\{\gamma_{i}\right\}^{\uparrow}\right\rangle \in \Delta_{w}$ for $1 \leq i \leq n$. Then, $\langle p, w\rangle$ is covered by $\left\langle p^{\prime}, w^{\prime}\right\rangle$ in $\mathcal{P}$ if and only if $w^{\prime} \in \delta_{\mathcal{P}^{\prime}, \mathcal{A}_{w}}\left(p, \#^{m}, p^{\prime}\right)$ where $m=\left|w^{\prime}\right|$. 
7.4. Pushdown Systems with Stack Manipulation. Uezato and Minamide introduced pushdown systems with stack manipulation (TrPDS) that can modify the whole stack content with a letter-to-letter finite-state transducer at each transition [UM13. TrPDS generalizes conditional pushdown systems [EKS03, LO10] and discrete timed pushdown systems AAS12. They showed that the reachability problem of a TrPDS is decidable if the closure of transductions appearing in the transition rules is finite.

The behaviour of a letter-to-letter transducer whose input and output alphabets are $\Gamma$ is characterized by a regular language over $\Gamma \times \Gamma$. Thus, we identify a letter-to-letter transducer with a corresponding regular language over $\Gamma \times \Gamma$ and call it a transduction. Let $w=a_{1} a_{2} \cdots a_{n}$ and $w^{\prime}=b_{1} b_{2} \cdots b_{n}$. We abuse the tuple notation and write $\left\langle w, w^{\prime}\right\rangle$ for $\left\langle a_{1}, b_{1}\right\rangle\left\langle a_{1}, b_{1}\right\rangle \cdots\left\langle a_{n}, b_{n}\right\rangle$ if it is clear from the context. For a transduction $t$, the left quotient of the transduction is defined as follows: $\left\langle\gamma_{1}, \gamma_{2}\right\rangle^{-1} t=\left\{\left\langle w_{1}, w_{2}\right\rangle \mid\left\langle\gamma_{1} w_{1}, \gamma_{2} w_{2}\right\rangle \in t\right\}$.

We say that $\mathcal{T} \subseteq \operatorname{Reg}(\Gamma \times \Gamma)$ is closed if the following hold.

- $\varnothing \in \mathcal{T}$ and $\left\{\langle w, w\rangle \mid w \in \Gamma^{*}\right\} \in \mathcal{T}$.

- If $t_{1}, t_{2} \in \mathcal{T}$, then $t_{1} \circ t_{2} \in \mathcal{T}$ and $t_{1} \cup t_{2} \in \mathcal{T}$.

- If $t \in \mathcal{T}$, then $\left\langle\gamma_{1}, \gamma_{2}\right\rangle^{-1} t \in \mathcal{T}$ for all $\gamma_{1}, \gamma_{2} \in \Gamma$.

We sometimes write $0_{\mathcal{T}}$ and $1_{\mathcal{T}}$ for $\varnothing$ and $\left\{\langle w, w\rangle \mid w \in \Gamma^{*}\right\}$, respectively.

Definition 7.5. A $\operatorname{TrPDS} \mathcal{P}$ is a structure $\langle P, \Gamma, \mathcal{T}, \Delta\rangle$ where $P$ is a finite set of states, $\Gamma$ is a stack alphabet, $\mathcal{T} \subseteq \operatorname{Reg}(\Gamma \times \Gamma)$ is a finite, closed set of transductions, and $\Delta \subseteq$ $P \times \Gamma \times P \times \Gamma^{*} \times \mathcal{T}$ is a set of transitions.

We write $\langle p, \gamma\rangle \stackrel{t}{\hookrightarrow}\left\langle p^{\prime}, w\right\rangle$ if $\left\langle p, \gamma, p^{\prime}, w, t\right\rangle \in \Delta$ as weighted pushdown systems. The transition relation of a TrPDS is defined as follows.

- $\langle p, w\rangle \Rightarrow\langle p, w\rangle$.

- $\left\langle p, \gamma w^{\prime}\right\rangle \Rightarrow\left\langle p^{\prime}, w w^{\prime \prime}\right\rangle$ if $\langle p, \gamma\rangle \stackrel{t}{\hookrightarrow}\left\langle p^{\prime}, w\right\rangle$ and $\left\langle w^{\prime}, w^{\prime \prime}\right\rangle \in t$.

- $\langle p, w\rangle \Rightarrow\left\langle p^{\prime}, w^{\prime}\right\rangle$ if $\langle p, w\rangle \Rightarrow\left\langle p^{\prime \prime}, w^{\prime \prime}\right\rangle$ and $\left\langle p^{\prime \prime}, w^{\prime \prime}\right\rangle \Rightarrow\left\langle p^{\prime}, w^{\prime}\right\rangle$.

In the second case above, the stack content below the top is modified by the transduction $t$.

A TrPDS can be simulated by combining the ideas of simulations in Section 7.1 and 7.2 , We again use the singleton stack alphabet $\Gamma^{\prime}=\{\#\}$ and define weight structure $\left\langle\left\{D_{\sigma}\right\}\right.$, $\left.\left\{\oplus_{\sigma}\right\},\left\{\odot_{\sigma_{1}, \sigma_{2}}\right\},\left\{0_{\sigma}\right\},\left\{1_{\sigma}\right\},\left\{\uparrow_{\sigma_{1}, \sigma_{2}}\right\}\right\rangle$ as follows.

- $D_{m / n}=\Gamma^{m} \times \Gamma^{n} \rightarrow \mathcal{T}$.

- $0_{m / n}\left(w_{1}, w_{2}\right)=0_{\mathcal{T}}$ and

$$
1_{m / m}\left(w_{1}, w_{2}\right)= \begin{cases}1_{\mathcal{T}} & \left(\text { if } w_{1}=w_{2}\right) \\ 0_{\mathcal{T}} & \text { (otherwise) }\end{cases}
$$

- For $f_{1} \in \Gamma^{l} \times \Gamma^{m} \rightarrow \mathcal{T}$ and $f_{2} \in \Gamma^{m} \times \Gamma^{n} \rightarrow \mathcal{T}, f_{1} \odot_{l / m, m / n} f_{2}$ is defined by

$$
\lambda\left(w_{1}, w_{3}\right) . \bigcup_{w_{2} \in \Gamma^{m}} f_{1}\left(w_{1}, w_{2}\right) \circ f_{2}\left(w_{2}, w_{3}\right) .
$$

- For $f_{1}, f_{2} \in \Gamma^{m} \times \Gamma^{n} \rightarrow \mathcal{T}, f_{1} \oplus_{m / n} f_{2}$ is defined by

$$
\lambda\left(w_{1}, w_{2}\right) . f_{1}\left(w_{1}, w_{2}\right) \cup f_{2}\left(w_{1}, w_{2}\right) .
$$

- $\uparrow_{l / m, l+1 / m+1}$ extends the domain of a function and is defined by

$$
\uparrow_{l / m, l+1 / m+1}(f)\left(w_{1} \gamma_{1}, w_{2} \gamma_{2}\right)=\left\langle\gamma_{1}, \gamma_{2}\right\rangle^{-1} f\left(w_{1}, w_{2}\right) .
$$


This structure forms a weight structure, and induces a locally bounded indexed semiring because $\mathcal{T}$ is a finite set.

We simulate a $\operatorname{TrPDS} \mathcal{P}=\langle P, \Gamma, \Delta\rangle$ by a weighted pushdown system $\mathcal{P}^{\prime}=\left\langle P,\{\#\}, \Delta^{\prime}\right\rangle$. For a transduction $t \in \mathcal{T}$, we define the function $t_{\gamma, w}: \Gamma \times \Gamma^{|w|} \rightarrow \mathcal{T}$ as follows.

$$
t_{\gamma, w}\left(\gamma^{\prime}, w^{\prime}\right)= \begin{cases}t & \text { if } \gamma^{\prime}=\gamma \text { and } w^{\prime}=w \\ 0 \mathcal{T} & \text { otherwise }\end{cases}
$$

Then, $\Delta^{\prime}$ is given by

$$
\left\langle p, \#, p^{\prime}, \#^{|w|}, t_{\gamma, w}\right\rangle \in \Delta^{\prime} \quad \text { iff } \quad\left\langle p, \gamma, p^{\prime}, w, t\right\rangle \in \Delta .
$$

$\mathcal{P}$ is simulated by $\mathcal{P}^{\prime}$ in the following sense. Hence, the reachability in $\mathcal{P}$ can be decided by the reachability analysis in $\mathcal{P}^{\prime}$. The proof of the following proposition appears in Appendix $\mathrm{C}$.

Proposition 7.6. Let $m_{1}=\left|w_{1}\right|$ and $m_{2}=\left|w_{2}\right|$.

- If $\left\langle p_{1}, w_{1}\right\rangle \underset{\mathcal{P}}{\Rightarrow}\left\langle p_{2}, w_{2}\right\rangle$, then $\left\langle p_{1}, m_{1}\right\rangle \underset{\mathcal{P}^{\prime}}{\stackrel{a}{\Rightarrow}}\left\langle p_{2}, m_{2}\right\rangle$ and $\langle\epsilon, \epsilon\rangle \in a\left(w_{1}, w_{2}\right)$ for some a.

- If $\left\langle p_{1}, m_{1}\right\rangle \underset{\mathcal{P}^{\prime}}{\vec{a}}\left\langle p_{2}, m_{2}\right\rangle$ and $\langle\epsilon, \epsilon\rangle \in a\left(w_{1}, w_{2}\right)$, then $\left\langle p_{1}, w_{1}\right\rangle \underset{\mathcal{P}}{\Rightarrow}\left\langle p_{2}, w_{2}\right\rangle$.

The backward reachability analysis similar to the above was presented by Uezato and Minamide [UM13. However, they used an ad-hoc extension of automata to generalize the saturation procedure and their presentation was rather complicated. We here greatly clarify the presentation by using our framework of weighted pushdown systems.

\section{Related Work}

An automaton over a monoid $\mathcal{M}$ is called a generalized $\mathcal{M}$-automaton by Eilenberg [Eil74]. The textbook of Sakarovitch discusses automata over several classes of monoids including free groups and commutative monoids [Sak09]. As far as we know, this paper is the first work that discusses the reachability analysis of pushdown systems by considering them as automata over the monoid of stack signatures.

Let us consider a paired alphabet $\widetilde{\Gamma}=\Gamma \cup \bar{\Gamma}$ where $\bar{\Gamma}=\{\bar{a} \mid a \in \Gamma\}$. Letters $\gamma$ and $\bar{\gamma}$ correspond to a push and a pop of $\gamma$, respectively. Then, the monoid $\mathcal{M}_{\Gamma}$ is closely related to the monoid over $\widetilde{\Gamma}^{*}$ obtained by Shamir congruence [Sha67], which is generated by $\gamma \bar{\gamma}=\epsilon$. If we add the relation $\gamma \overline{\gamma^{\prime}}=\top$ for $\gamma \neq \gamma^{\prime}$, then the reduced form of a word over $\widetilde{\Gamma}$ has the following form: $\overline{w_{1}} w_{2}$ or $\top$. If we write $w_{1} / w_{2}^{R}$ for $\overline{w_{1}} w_{2}$, we obtain a stack signature?.

Esparza et al. showed that conditional pushdown systems can be translated to ordinary pushdown systems [EKS03]. Hence, the reachability can be decided via the translation. However, it is not practical to apply the translation because of exponential blowup of the size of pushdown systems. The algorithm formulated in Section 7.2 as the reachability analysis of weighted pushdown systems has also an exponential complexity. However, it avoids the exponential blowup by the translation before applying the reachability analysis and worked well for the analysis of the HTML5 parser specification [MM12].

Reps et al. [RSJM05] developed both of the forward and backward analysis of weighted pushdown systems. Although our backward analysis is a direct extension of their analysis, the forward reachability analysis cannot directly be extended for indexed weight domains. This is because $a \in D_{\gamma / \gamma^{\prime} \gamma^{\prime \prime}}$ cannot be decomposed to $a=a_{1} \otimes a_{2}$ for $a_{1} \in D_{\gamma / \gamma^{\prime \prime}}$ and $a_{2} \in D_{\epsilon / \gamma^{\prime}}$ in general. If this decomposition is possible, a slightly modified version of their

\footnotetext{
${ }^{7} w_{2}{ }^{R}$ is the reverse of $w_{2}$.
} 
forward reachability analysis can be extended for indexed weighted domains (we add a new states $q_{r}$ indexed by a transition rule $r$ as the original forward reachability analysis considered by Esparza et.al [EHRS00] instead of $q_{p^{\prime}, \gamma^{\prime}}$ indexed by a state $p^{\prime}$ and a pushdown symbol $\gamma^{\prime}$.). However, among the four indexed semirings in Section 7, only the indexed semiring for conditional pushdown systems enables the decomposition above. It should be noted that Cai and Ogawa developed the forward reachability analysis of well-structured pushdown systems by combining the saturation procedure with the Karp-Miller acceleration instead of the ideal representation [CO13].

\section{Conclusions}

We have introduced the monoid of stack signatures to treat pushdown systems as automata over the monoid. Then, weighted pushdown systems are generalized by adopting a semiring indexed by stack signatures as weight. This generalization makes it possible to relax the restriction of boundedness and extend the applications of the reachability analysis of weighted pushdown systems.

We have shown that by designing proper indexed semirings, the reachability analysis of several extensions of pushdown systems can be achieved by a translation to weighted pushdown systems and their reachability analysis. Although the reachability analysis of those extensions were already developed by directly extending the analysis of ordinary pushdown systems, our approach clarifies the analysis by separating the design of indexed semirings, which depends on each extension, from the general algorithm of the reachability analysis.

The indexed semirings for the applications in this paper are given through weight structures. We consider that it is simpler to construct and implement indexed semirings through weight structures than to directly construct them. However, we are not completely satisfied with the formulation of weight structures because their definition looks rather ad-hoc mathematically. We would like to investigate more abstract notion corresponding to weight structures.

\section{ACKNOWLEDGMENTS}

I would like to thank Stefan Schwoon for inspiring discussions and suggestions. Schwoon informed me of the work of Suwimonteerabuth on the encoding of local variables into weight. I would also like to thank Shin-ya Katsumata for his comments on lax monoidal functors and graded semirings. The paper has also benefited from constructive feedback and suggestions by the anonymous referees, which are greatly appreciated. This work has been partially supported by JSPS Grant-in-Aid for Science Research (C) 24500028 and 15K00087, and the Kayamori Foundation of Informational Science Advancement.

\section{REFERENCES}

[AAS12] Parosh Aziz Abdulla, Mohamed Faouzi Atig, and Jari Stenman. The minimal cost reachability problem in priced timed pushdown systems. In Language and Automata Theory and Applications - 6th International Conference, LATA 2012, Proceedings, pages 58-69, 2012. LNCS 7183.

[BEM97] A. Bouajjani, J. Esparza, and O. Maler. Reachability analysis of pushdown automata: application to model-checking. In CONCUR '97, pages 135-150, 1997. LNCS 1243. 
[CO13] Xiaojuan Cai and Mizuhito Ogawa. Well-structured pushdown systems. In CONCUR 2013 Concurrency Theory, pages 121-136, 2013. LNCS 8052.

[EHRS00] Javier Esparza, David Hansel, Peter Rossmanith, and Stefan Schwoon. Efficient algorithms for model checking pushdown systems. In E. Allen Emerson and A. Prasad Sistla, editors, Proceedings of the 12th International Conference on Computer Aided Verification (CAV 2000), volume 1855 of Lecture Notes in Computer Science, pages 232-247. Springer, 2000.

[Eil74] Samuel Eilenberg. Automata, Languages, and Machines, volume A. Academic Press, 1974.

[ÉK09] Zoltán Ésik and Werner Kuich. Finite automata. In Manfred Droste, Werner Kuich, and Heiko Vogler, editors, Handbook of Weighted Automata, chapter 3, pages 69-104. Springer, 2009.

[EKS03] J. Esparza, A. Kucera, and S. Schwoon. Model checking LTL with regular valuations for pushdown systems. Information and Computation, 186(2):355-376, 2003.

[FS01] Alain Finkel and Philippe Schnoebelen. Well-structured transition systems everywhere! Theoretical Computer Science, 256(1-2):63-92, April 2001.

[FWW97] A. Finkel, B. Willems, and P. Wolper. A direct symbolic approach to model checking pushdown systems. In INFINITY '97, pages 27-39, 1997. ENTCS 9.

[Lan02] Serge Lang. Algebra, volume 211 of Graduate Texts in Mathematics. Springer-Verlag, 2002.

[LO10] Xin Li and Mizuhito Ogawa. Conditional weighted pushdown systems and applications. In Proceedings of the 2010 ACM SIGPLAN Workshop on Partial Evaluation and Program Manipulation, pages 141-150, 2010.

[Min07] Yasuhiko Minamide. Verified decision procedures on context-free grammars. In Proc. of the 20th International Conference Theorem Proving in Higher Order Logics, pages 173-188, 2007. LNCS 4732 .

[MM12] Yasuhiko Minamide and Shunsuke Mori. Reachability analysis of the HTML5 parser specification and its application to compatibility testing. In Proceedings of the 18th International Symposium on Formal Methods, volume 7436 of LNCS, pages 293-307, 2012.

[MT06] Yasuhiko Minamide and Akihiko Tozawa. XML validation for context-free grammars. In Proc. of The Fourth ASIAN Symposium on Programming Languages and Systems, volume 4279 of LNCS, pages 357-373, 2006.

[RSJM05] Thomas Reps, Stefan Schwoon, Somesh Jha, and David Melski. Weighted pushdown systems and their application to interprocedural dataflow analysis. Science of Computer Programming, 58:206-263, 2005.

[Sak09] Jacques Sakarovitch. Elements of Automata Theory. Cambridge University Press, 2009.

[Sha67] Eliahu Shamir. A representation theorem for algebraic and context-free power series in non commuting variables. Information and Control, 11(1/2):239-254, 1967.

[Suw09] Dejvuth Suwimonteerabuth. Reachability in Pushdown Systems: Algorithms and Applications. PhD thesis, Technischen Universität München, 2009.

[TM07] Akihiko Tozawa and Yasuhiko Minamide. Complexity results on balanced context-free languages. In Proc. of Tenth International Conference on Foundations of Software Science and Computation Structures, volume 4423 of LNCS, pages 346-360, 2007.

[UM13] Yuya Uezato and Yasuhiko Minamide. Pushdown systems with stack manipulation. In Proc. of 11th International Symposium on Automated Technology for Verification and Analysis (ATVA), pages 412-426, 2013. LNCS 8172.

\section{Appendix A. Proofs on Stack Signatures}

Lemma A.1. $\left(w_{1} / w_{1}^{\prime} \cdot w_{2} / w_{2}^{\prime}\right) \cdot w_{3} / w_{3}^{\prime}=w_{1} / w_{1}^{\prime} \cdot\left(w_{2} / w_{2}^{\prime} \cdot w_{3} / w_{3}^{\prime}\right)$

Proof. By case analysis on the prefix relation. We omit the cases where $\left(w_{1} / w_{1}^{\prime} \cdot w_{2} / w_{2}^{\prime}\right)$. $w_{3} / w_{3}^{\prime}=w_{1} / w_{1}^{\prime} \cdot\left(w_{2} / w_{2}^{\prime} \cdot w_{3} / w_{3}^{\prime}\right)=\top$.

(1) $w_{1}^{\prime}$ is a prefix of $w_{2}$, i.e., $w_{2}=w_{1}^{\prime} w_{2}^{\prime \prime}$. 
(a) $w_{2}^{\prime}$ is a prefix of $w_{3}$, i.e., $w_{3}=w_{2}^{\prime} w_{3}^{\prime \prime}$.

$$
\begin{aligned}
\left(w_{1} / w_{1}^{\prime} \cdot w_{2} / w_{2}^{\prime}\right) \cdot w_{3} / w_{3}^{\prime} & =w_{1} w_{2}^{\prime \prime} / w_{2}^{\prime} \cdot w_{3} / w_{3}^{\prime} \\
& =w_{1} w_{2}^{\prime \prime} w_{3}^{\prime \prime} / w_{3}^{\prime} \\
& =w_{1} / w_{1}^{\prime} \cdot w_{1}^{\prime} w_{2}^{\prime \prime} w_{3}^{\prime \prime} / w_{3}^{\prime} \\
& =w_{1} / w_{1}^{\prime} \cdot\left(w_{2} / w_{2}^{\prime} \cdot w_{3} / w_{3}^{\prime}\right)
\end{aligned}
$$

(b) $w_{3}$ is a prefix of $w_{2}^{\prime}$, i.e., $w_{2}^{\prime}=w_{3} w_{2}^{\prime \prime \prime}$.

$$
\begin{aligned}
\left(w_{1} / w_{1}^{\prime} \cdot w_{2} / w_{2}^{\prime}\right) \cdot w_{3} / w_{3}^{\prime} & =w_{1} w_{2}^{\prime \prime} / w_{2}^{\prime} \cdot w_{3} / w_{3}^{\prime} \\
& =w_{1} w_{2}^{\prime \prime} / w_{3}^{\prime} w_{2}^{\prime \prime \prime} \\
& =w_{1} / w_{1}^{\prime} \cdot w_{2} / w_{3}^{\prime} w_{2}^{\prime \prime \prime} \\
& =w_{1} / w_{1}^{\prime} \cdot\left(w_{2} / w_{2}^{\prime} \cdot w_{3} / w_{3}^{\prime}\right)
\end{aligned}
$$

(2) $w_{2}$ is a prefix of $w_{1}^{\prime}$, i.e., $w_{1}^{\prime}=w_{2} w_{1}^{\prime \prime}$.

(a) $w_{2}^{\prime}$ is a prefix of $w_{3}$, i.e., $w_{3}=w_{2}^{\prime} w_{3}^{\prime \prime}$.

(i) $w_{1}^{\prime \prime}$ is a prefix of $w_{3}^{\prime \prime}$, i.e., $w_{3}^{\prime \prime}=w_{1}^{\prime \prime} w$.

$$
\begin{aligned}
\left(w_{1} / w_{1}^{\prime} \cdot w_{2} / w_{2}^{\prime}\right) \cdot w_{3} / w_{3}^{\prime} & =w_{1} / w_{2}^{\prime} w_{1}^{\prime \prime} \cdot w_{2}^{\prime} w_{3}^{\prime \prime} / w_{3}^{\prime} \\
& =w_{1} w / w_{3}^{\prime} \\
& =w_{1} / w_{2} w_{1}^{\prime \prime} \cdot w_{2} w_{3}^{\prime \prime} / w_{3}^{\prime} \\
& =w_{1} / w_{1}^{\prime} \cdot\left(w_{2} / w_{2}^{\prime} \cdot w_{3} / w_{3}^{\prime}\right)
\end{aligned}
$$

(ii) $w_{3}^{\prime \prime}$ is a prefix of $w_{1}^{\prime \prime}$. Symmetric to the case above.

(b) $w_{3}$ is a prefix of $w_{2}^{\prime}$, i.e., $w_{2}^{\prime}=w_{3} w_{2}^{\prime \prime \prime}$. This case is symmetric to Case (1a).

Lemma A.2. If $\sigma_{1} \cdot \sigma_{2} \cdot \sigma_{3} \neq \top$, one of the followings holds.

(1) $\sigma_{1} \leq \sigma_{1}^{\prime}, \sigma_{3} \leq \sigma_{3}^{\prime}, \sigma_{1}^{\prime} \| \sigma_{2}$, and $\sigma_{2} \| \sigma_{3}^{\prime}$.

(2) $\sigma_{1} \leq \sigma_{1}^{\prime}, \sigma_{2} \leq \sigma_{2}^{\prime}, \sigma_{1}^{\prime} \| \sigma_{2}$, and $\sigma_{2}^{\prime} \| \sigma_{3}$.

(3) $\sigma_{3} \leq \sigma_{3}^{\prime}, \sigma_{2} \leq \sigma_{2}^{\prime}, \sigma_{2} \| \sigma_{3}^{\prime}$, and $\sigma_{1} \| \sigma_{2}^{\prime}$.

(4) $\sigma_{2} \leq \sigma_{2}^{\prime} \leq \sigma_{2}^{\prime \prime}, \sigma_{1} \| \sigma_{2}^{\prime}$, and $\sigma_{2}^{\prime \prime} \| \sigma_{3}$.

(5) $\sigma_{2} \leq \sigma_{2}^{\prime} \leq \sigma_{2}^{\prime \prime}, \sigma_{1} \| \sigma_{2}^{\prime \prime}$, and $\sigma_{2}^{\prime} \| \sigma_{3}$.

Proof. This lemma is obtained by inspecting the proof of the above lemma.

Lemma A.3. If $\sigma_{1} \leq \sigma_{1}^{\prime}$ and $\sigma_{2} \leq \sigma_{2}^{\prime}$, then $\sigma_{1} \cdot \sigma_{2} \leq \sigma_{1}^{\prime} \cdot \sigma_{2}^{\prime}$.

Proof. It is sufficient to prove the proposition for the case $\sigma_{1}^{\prime} \cdot \sigma_{2}^{\prime} \neq \top$. Then, there exist strictly compatible $\sigma_{1}^{\prime \prime}$ and $\sigma_{2}^{\prime \prime}$ such that $\sigma_{1}^{\prime} \leq \sigma_{1}^{\prime \prime}, \sigma_{2}^{\prime} \leq \sigma_{2}^{\prime \prime}$, and $\sigma_{1}^{\prime} \cdot \sigma_{2}^{\prime}=\sigma_{1}^{\prime \prime} \cdot \sigma_{2}^{\prime \prime}$. Thus, we can assume that $\sigma_{1}^{\prime}$ and $\sigma_{2}^{\prime}$ are strictly compatible.

Case $\sigma_{1} \cdot \sigma_{2} \neq \top$ : Without loss of generality, we assume that $\sigma_{1}=w_{1} / w$ and $\sigma_{2}=w w_{2} / w_{2}^{\prime}$.

Then, we have $\sigma_{1}^{\prime}=w_{1} w_{2} w^{\prime} / w w_{2} w^{\prime}$ and $\sigma_{2}^{\prime}=w w_{2} w^{\prime} / w_{2}^{\prime} w^{\prime}$ for some $w^{\prime}$. Hence, $w_{1} w_{2} / w_{2}^{\prime}=\sigma_{1} \cdot \sigma_{2} \leq \sigma_{1}^{\prime} \cdot \sigma_{2}^{\prime}=w_{1} w_{2} w^{\prime} / w_{2}^{\prime} w^{\prime}$.

Case $\sigma_{1} \cdot \sigma_{2}=\top$ : This case contradicts $\sigma_{1}^{\prime} \cdot \sigma_{2}^{\prime} \neq \top$.

Lemma A.4. Let $\sigma \neq \top$. If $\sigma_{1} \leq \sigma$ and $\sigma_{2} \leq \sigma$, then either $\sigma_{1} \leq \sigma_{2}$ or $\sigma_{2} \leq \sigma_{1}$.

Proof. This lemma can be easily proved by case analysis. 
Lemma A.5. $\left(\sigma_{1} \sqcup \sigma_{2}\right) \cdot \sigma_{3}=\left(\sigma_{1} \cdot \sigma_{3}\right) \sqcup\left(\sigma_{2} \cdot \sigma_{3}\right)$.

Proof. If $\sigma_{1} \leq \sigma_{2}$, then $\sigma_{1} \cdot \sigma_{3} \leq \sigma_{2} \cdot \sigma_{3}$ by Lemma A.3 and thus the proposition holds. To cover the other case, we show $\sigma_{1} \sqcup \sigma_{2} \neq \top$ by assuming $\left(\sigma_{1} \cdot \sigma_{3}\right) \sqcup\left(\sigma_{2} \cdot \sigma_{3}\right) \neq \top$.

Case 1: $\sigma_{1} \cdot \sigma_{3}=\sigma_{1} \cdot \sigma_{3}^{\prime}$ for strictly compatible $\sigma_{1}$ and $\sigma_{3}^{\prime}$, and $\sigma_{2} \cdot \sigma_{3}=\sigma_{2} \cdot \sigma_{3}^{\prime \prime}$ for strictly compatible $\sigma_{2}$ and $\sigma_{3}^{\prime \prime}$. By Lemma A.4, without loss of generality, we assume $\sigma_{1} \cdot \sigma_{3} \leq \sigma_{2} \cdot \sigma_{3}$

Let $\sigma_{1}=w_{1} / w_{1}^{\prime}, \sigma_{2}=w_{2} / w_{2}^{\prime}$, and $\sigma_{3}=w_{3} / w_{3}^{\prime}$. Then, $w_{1}^{\prime}=w_{3} w_{13}$ and $w_{2}^{\prime}=w_{3} w_{23}$ for some $w_{13}$ and $w_{23}$. Then, $\sigma_{1} \cdot \sigma_{3}=w_{1} / w_{3}^{\prime} w_{13}$ and $\sigma_{2} \cdot \sigma_{3}=w_{2} / w_{3}^{\prime} w_{23}$. From $\sigma_{1} \cdot \sigma_{3} \leq \sigma_{2} \cdot \sigma_{3}, w_{2}=w_{1} w$ and $w_{23}=w_{13} w$ for some $w$. Then, $\sigma_{1}=w_{1} / w_{3} w_{13}$ and $\sigma_{2}=w_{1} w / w_{3} w_{13} w$

Case 2: $\sigma_{1} \cdot \sigma_{3}=\sigma_{1} \cdot \sigma_{3}^{\prime}$ for strictly compatible $\sigma_{1}$ and $\sigma_{3}^{\prime}$, and $\sigma_{2} \cdot \sigma_{3}=\sigma_{2}^{\prime} \cdot \sigma_{3}$ for strictly compatible $\sigma_{2}^{\prime}$ and $\sigma_{3}$. Let $\sigma_{1}=w_{1} / w_{1}^{\prime}, \sigma_{2}=w_{2} / w_{2}^{\prime}$, and $\sigma_{3}=w_{3} / w_{3}^{\prime}$. Then, $w_{1}^{\prime}=w_{3} w_{13}$ and $w_{3}=w_{2}^{\prime} w_{23}$ for some $w_{13}$ and $w_{23}$. Then, $\sigma_{1} \cdot \sigma_{3}=w_{1} / w_{3}^{\prime} w_{13}$ and $\sigma_{2} \cdot \sigma_{3}=w_{2} w_{23} / w_{3}^{\prime}$.

- Subcase $\sigma_{2} \cdot \sigma_{3} \leq \sigma_{1} \cdot \sigma_{3}$. Then, we have $w_{1}=w_{2} w_{23} w_{13}$ hence $\sigma_{1}=w_{2} w_{23} w_{13} / w_{2}^{\prime} w_{23} w_{13}$ and therefore $\sigma_{2}=w_{2} / w_{2}^{\prime}$.

- Subcase $\sigma_{1} \cdot \sigma_{3}<\sigma_{2} \cdot \sigma_{3}$. This case does not occur because $\sigma_{1} \cdot \sigma_{3}=w_{1} / w_{3}^{\prime} w_{13}$ and $\sigma_{2} \cdot \sigma_{3}=w_{2} w_{23} / w_{3}^{\prime}$.

Case 3: $\sigma_{1} \cdot \sigma_{3}=\sigma_{1}^{\prime} \cdot \sigma_{3}$ for strictly compatible $\sigma_{1}^{\prime}$ and $\sigma_{3}$, and $\sigma_{2} \cdot \sigma_{3}=\sigma_{2}^{\prime} \cdot \sigma_{3}$ for strictly compatible $\sigma_{2}^{\prime}$ and $\sigma_{3}$. From $\left(\sigma_{1} \cdot \sigma_{3}\right) \sqcup\left(\sigma_{2} \cdot \sigma_{3}\right) \neq \top$, we have $\sigma_{1}^{\prime} \cdot \sigma_{3}=\sigma_{2}^{\prime} \cdot \sigma_{3}$. Then, $\sigma_{1}^{\prime}=\sigma_{2}^{\prime}$. Hence, we have $\sigma_{1} \leq \sigma_{2}$ or $\sigma_{2} \leq \sigma_{1}$ by Lemma A.4.

Case 4: $\sigma_{1} \cdot \sigma_{3}=\sigma_{1}^{\prime} \cdot \sigma_{3}$ for strictly compatible $\sigma_{1}^{\prime}$ and $\sigma_{3}$, and $\sigma_{2} \cdot \sigma_{3}=\sigma_{2} \cdot \sigma_{3}^{\prime}$ for strictly compatible $\sigma_{2}$ and $\sigma_{3}^{\prime}$. This case is the same as the case 2 by exchanging $\sigma_{1}$ and $\sigma_{2}$.

\section{Appendix B. Correspondence for Well-Structured Pushdown Systems}

\section{Restatement of Proposition 7.4.}

- If $\left\langle p_{1}, w_{1}\right\rangle \underset{\mathcal{P}}{\Rightarrow}\left\langle p_{2}, w_{2}\right\rangle$, then $\left\langle p_{1}, m_{1}\right\rangle \underset{\mathcal{P}^{\prime}}{\stackrel{a}{\Rightarrow}}\left\langle p_{2}, m_{2}\right\rangle$ and $w_{1} \in a\left(w_{2}\right)$.

- If $\left\langle p_{1}, m_{1}\right\rangle \underset{\mathcal{P}^{\prime}}{\stackrel{a}{\Rightarrow}}\left\langle p_{2}, m_{2}\right\rangle$ and $w_{1} \in a\left(w_{2}\right)$, then $\left\langle p_{1}, w_{1}\right\rangle \underset{\mathcal{P}}{\Rightarrow}\left\langle p_{2}, w_{2}^{\prime}\right\rangle$ for some $w_{2} \preceq w_{2}^{\prime}$.

where $m_{1}=\left|w_{1}\right|$ and $m_{2}=\left|w_{2}\right|$.

Proof.

- We prove the first statement by induction on the derivation of $\left\langle p_{1}, w_{1}\right\rangle \underset{\mathcal{P}}{\Rightarrow}\left\langle p_{2}, w_{2}\right\rangle$.

Case: $\langle p, w\rangle \Rightarrow\langle p, w\rangle$ where $|w|=m$. Then, $\langle p, m\rangle \underset{\overrightarrow{\mathcal{P}^{\prime}}}{\stackrel{a}{\Rightarrow}}\langle p, m\rangle$ where $\left.a=\lambda w \cdot\{w\}^{\uparrow}\right)$. Then, $w \in a(w)$.

Case: $\left\langle p, \gamma w^{\prime}\right\rangle \Rightarrow\left\langle p^{\prime}, \phi(\gamma) w^{\prime}\right\rangle,\left|w^{\prime}\right|=m$, and $|\phi(\gamma)|=i$. Then, $\left\langle p_{1}, m+1\right\rangle \underset{\overrightarrow{\mathcal{P}^{\prime}}}{\vec{a}}\left\langle p_{2}, m+i\right\rangle$ where $a=\lambda\left\langle w, w^{\prime}\right\rangle \cdot \phi^{-1}\left(\{w\}^{\uparrow}\right) \times\left\{w^{\prime}\right\}^{\uparrow}$. Then, we have $a\left(\phi(\gamma) w^{\prime}\right)=\phi^{-1}\left(\{\phi(\gamma)\}^{\uparrow}\right) \times$ $\left\{w^{\prime}\right\}^{\uparrow} \ni \gamma w^{\prime}$.

Case: $\left\langle p_{1}, w_{1}\right\rangle \underset{\mathcal{P}}{\Rightarrow}\left\langle p_{3}, w_{3}\right\rangle$ is obtained from $\left\langle p_{1}, w_{1}\right\rangle \underset{\mathcal{P}}{\Rightarrow}\left\langle p_{2}, w_{2}\right\rangle$ and $\left\langle p_{2}, w_{2}\right\rangle \underset{\mathcal{P}}{\Rightarrow}\left\langle p_{3}, w_{3}\right\rangle$. By the induction hypotheses we have $\left\langle p_{1}, m_{1}\right\rangle \underset{\mathcal{P}^{\prime}}{\overrightarrow{a_{1}}}\left\langle p_{2}, m_{2}\right\rangle$ and $w_{1} \in a\left(w_{2}\right)$, as well as $\left\langle p_{2}, m_{2}\right\rangle \underset{\mathcal{P}^{\prime}}{\stackrel{a_{2}}{\Rightarrow}}\left\langle p_{3}, m_{3}\right\rangle$ and $w_{2} \in a\left(w_{3}\right)$. Then, $\left\langle p_{1}, m_{1}\right\rangle \stackrel{a_{1} \odot a_{2}}{\stackrel{\mathcal{P}^{\prime}}{\rightleftarrows}}\left\langle p_{2}, m_{2}\right\rangle$ and $a_{1} \odot a_{2}\left(w_{3}\right)=\bigcup_{w \in a_{2}\left(w_{3}\right)} a_{1}(w) \supseteq a_{1}\left(w_{2}\right) \ni w_{1}$. 
- We prove the second statement by induction on the derivation of $\left\langle p_{1}, m_{1}\right\rangle \underset{\mathcal{P}^{\prime}}{\Rightarrow}\left\langle p_{2}, m_{2}\right\rangle$.

Case: $\left\langle p, \#^{m}\right\rangle \stackrel{1_{m / m}}{\underset{\mathcal{P}^{\prime}}{\longrightarrow}}\left\langle p, \#^{m}\right\rangle$. Let $w_{1} \in\left\{w_{2}\right\}^{\uparrow}=1_{m / m}\left(w_{2}\right)$. Then, $\left\langle p, w_{1}\right\rangle \underset{\mathcal{P}}{\Rightarrow}\left\langle p, w_{1}\right\rangle$ and $w_{2} \preceq w_{1}$.

Case: $\left\langle p_{1}, \#^{m+1}\right\rangle \underset{\mathcal{P}^{\prime}}{\stackrel{a}{\Rightarrow}}\left\langle p_{2}, \#^{m+i}\right\rangle$ is obtained from the fact that $\left(p_{1}, p_{2}, \phi\right) \in \Delta$ and from $a=\lambda\left\langle w, w^{\prime}\right\rangle \cdot \phi^{-1}\left(\{w\}^{\uparrow}\right) \times\left\{w^{\prime}\right\}^{\uparrow}$. Let $w_{2}=w_{2}^{\prime} w_{2}^{\prime \prime}$ and $w_{1}=\gamma w_{1}^{\prime \prime}$ where $\left|w_{2}^{\prime}\right|=i$ and $\left|w_{1}^{\prime \prime}\right|=\left|w_{2}^{\prime \prime}\right|=m$. Let $\gamma \in \phi^{-1}\left(\left\{w_{2}^{\prime}\right\}^{\uparrow}\right)$ and $w_{1}^{\prime \prime} \in\left\{w_{2}^{\prime \prime}\right\}^{\uparrow}$. Then, $\phi(\gamma)=w_{2}^{\prime \prime \prime}$ for some $w_{2}^{\prime} \preceq w_{2}^{\prime \prime \prime}$.

Hence, $\left\langle p_{1}, \gamma w_{1}^{\prime \prime}\right\rangle \underset{\mathcal{P}}{\Rightarrow}\left\langle p_{2}, w_{2}^{\prime \prime \prime} w_{1}^{\prime \prime}\right\rangle$ and $w_{2}=w_{2}^{\prime} w_{1}^{\prime \prime} \preceq w_{2}^{\prime \prime \prime} w_{1}^{\prime \prime}$.

Case: $\left\langle p_{1}, m_{1}\right\rangle \stackrel{a_{1} \odot a_{2}}{\underset{\mathcal{P}^{\prime}}{\longrightarrow}}\left\langle p_{3}, m_{3}\right\rangle$ is obtained from transitions $\left\langle p_{1}, m_{1}\right\rangle \stackrel{a_{1}}{\overrightarrow{\mathcal{P}^{\prime}}}\left\langle p_{2}, m_{2}\right\rangle$ and $\left\langle p_{2}, m_{2}\right\rangle \stackrel{a_{2}}{\overrightarrow{\mathcal{P}^{\prime}}}\left\langle p_{3}, m_{3}\right\rangle$. Let $w_{1} \in a_{1} \odot a_{2}\left(w_{3}\right)=\bigcup_{w \in a_{2}\left(w_{3}\right)} a_{1}(w)$. Then, $w_{1} \in a_{1}\left(w_{2}\right)$ and $w_{2} \in a_{2}\left(w_{3}\right)$ for some $w_{2}$. By the induction hypothesis, $\left\langle p_{1}, w_{1}\right\rangle \underset{\mathcal{P}}{\Rightarrow}\left\langle p_{2}, w_{2}^{\prime}\right\rangle$ for some $w_{2} \preceq w_{2}^{\prime}$ and $\left\langle p_{2}, w_{2}\right\rangle \underset{\mathcal{P}}{\Rightarrow}\left\langle p_{2}, w_{3}^{\prime}\right\rangle$ for some $w_{3} \preceq w_{3}^{\prime}$. By the monotonicity of $\mathcal{P}$, $\left\langle p_{2}, w_{2}^{\prime}\right\rangle \underset{\mathcal{P}}{\Rightarrow}\left\langle p_{2}, w_{3}^{\prime \prime}\right\rangle$ for some $w_{3}^{\prime} \preceq w_{3}^{\prime \prime}$. Then, $\left\langle p_{1}, w_{1}\right\rangle \underset{\mathcal{P}}{\Rightarrow}\left\langle p_{3}, w_{3}^{\prime \prime}\right\rangle$ and $w_{3} \preceq w_{3}^{\prime \prime}$.

\section{Appendix C. Correspondence for Pushdown Systems with Stack Manipulation}

Restatement of Proposition 7.6. Let $m_{1}=\left|w_{1}\right|$ and $m_{2}=\left|w_{2}\right|$.

- If $\left\langle p_{1}, w_{1}\right\rangle \underset{\mathcal{P}}{\Rightarrow}\left\langle p_{2}, w_{2}\right\rangle$, then $\left\langle p_{1}, m_{1}\right\rangle \underset{\overrightarrow{\mathcal{P}^{\prime}}}{\stackrel{a}{\Rightarrow}}\left\langle p_{2}, m_{2}\right\rangle$ and $\langle\epsilon, \epsilon\rangle \in a\left(w_{1}, w_{2}\right)$ for some a.

- If $\left\langle p_{1}, m_{1}\right\rangle \underset{\overrightarrow{\mathcal{P}^{\prime}}}{\vec{a}}\left\langle p_{2}, m_{2}\right\rangle$ and $\langle\epsilon, \epsilon\rangle \in a\left(w_{1}, w_{2}\right)$, then $\left\langle p_{1}, w_{1}\right\rangle \underset{\mathcal{P}}{\Rightarrow}\left\langle p_{2}, w_{2}\right\rangle$.

Proof. Let $\left|w_{i}\right|=m_{i}$ for $1 \leq i \leq 3$ in this proof.

- We prove the first statement by induction on the derivation of $\left\langle p_{1}, w_{1}\right\rangle \underset{\mathcal{P}}{\Rightarrow}\left\langle p_{2}, w_{2}\right\rangle$.

Case: $\left\langle p_{1}, w_{1}\right\rangle \underset{\mathcal{P}}{\Rightarrow}\left\langle p_{1}, w_{1}\right\rangle$. We have $\left\langle p, m_{1}\right\rangle \stackrel{1_{m_{1} / m_{1}}}{\underset{\mathcal{P}^{\prime}}{\Longrightarrow}}\left\langle p, m_{1}\right\rangle$ and $1_{m_{1} / m_{1}}\left(w_{1}, w_{1}\right)=1_{\mathcal{T}} \ni$ $\langle\epsilon, \epsilon\rangle$.

Case: $\left\langle p, \gamma w^{\prime}\right\rangle \underset{\mathcal{P}}{\Rightarrow}\left\langle p^{\prime}, w w^{\prime \prime}\right\rangle$ is obtained from $\left\langle p, \gamma, p^{\prime}, w, t\right\rangle \in \Delta$ and $\left\langle w^{\prime}, w^{\prime \prime}\right\rangle \in t$. Let $|w|=$ $n$ and $\left|w^{\prime}\right|=\left|w^{\prime \prime}\right|=m$. Then, $\left\langle p_{1}, m+1\right\rangle \underset{\overrightarrow{\mathcal{P}^{\prime}}}{\stackrel{a}{\Rightarrow}}\left\langle p_{2}, m+n\right\rangle$ where $a=\uparrow_{1 / n, 1+m / n+m}$ $\left(t_{\gamma, w}\right)$ and $a\left(\gamma w^{\prime}, w w^{\prime \prime}\right)=\left\langle w^{\prime}, w^{\prime \prime}\right\rangle^{-1}\left(t_{\gamma, w}(\gamma, w)\right)=\left\langle w^{\prime}, w^{\prime \prime}\right\rangle^{-1} t \ni\langle\epsilon, \epsilon\rangle$.

Case: $\left\langle p_{1}, w_{1}\right\rangle \underset{\mathcal{P}}{\Rightarrow}\left\langle p_{3}, w_{3}\right\rangle$ is obtained from $\left\langle p_{1}, w_{1}\right\rangle \underset{\mathcal{P}}{\Rightarrow}\left\langle p_{2}, w_{2}\right\rangle$ and $\left\langle p_{2}, w_{2}\right\rangle \underset{\mathcal{P}}{\Rightarrow}\left\langle p_{3}, w_{3}\right\rangle$. By the induction hypotheses, $\left\langle p_{1}, m_{1}\right\rangle \stackrel{a_{1}}{\overrightarrow{\mathcal{P}^{\prime}}}\left\langle p_{2}, m_{2}\right\rangle,\left\langle p_{2}, m_{2}\right\rangle \stackrel{a_{2}}{\overrightarrow{\mathcal{P}^{\prime}}}\left\langle p_{3}, m_{3}\right\rangle,\langle\epsilon, \epsilon\rangle \in$ $a_{1}\left(w_{1}, w_{2}\right)$, and $\langle\epsilon, \epsilon\rangle \in a_{2}\left(w_{2}, w_{3}\right)$. Then, $\left\langle p_{1}, m_{1}\right\rangle \stackrel{a_{1} \odot a_{2}}{\underset{\mathcal{P}^{\prime}}{\longrightarrow}}\left\langle p_{3}, m_{3}\right\rangle$ and $\langle\epsilon, \epsilon\rangle \in$ $a_{1}\left(w_{1}, w_{2}\right) \circ a_{2}\left(w_{2}, w_{3}\right) \subseteq a_{1} \odot a_{2}\left(w_{1}, w_{3}\right)$.

- We prove the second statement by induction on the derivation of $\left\langle p_{1}, m_{1}\right\rangle \underset{\mathcal{P}^{\prime}}{\stackrel{a}{\Rightarrow}}\left\langle p_{2}, m_{2}\right\rangle$.

Case: $\langle p, m\rangle \stackrel{1_{m / m}}{\underset{\mathcal{P}^{\prime}}{\Longrightarrow}}\langle p, m\rangle$ and $\langle\epsilon, \epsilon\rangle \in 1_{m / m}\left(w_{1}, w_{2}\right)$. By the definition of $1_{m / m}, w_{1}=w_{2}$. Thus, $\left\langle p, w_{1}\right\rangle \underset{\mathcal{P}}{\Rightarrow}\left\langle p, w_{2}\right\rangle$. 
Case: $\left\langle p_{1}, m+1\right\rangle \underset{\overrightarrow{\mathcal{P}^{\prime}}}{\stackrel{a}{\Rightarrow}}\left\langle p_{2}, m+n\right\rangle$ where $a=\uparrow_{1 / n, 1+m / n+m}\left(t_{\gamma, w}\right)$. Let $\langle\epsilon, \epsilon\rangle \in a\left(\gamma_{0} w^{\prime}, w_{0} w^{\prime \prime}\right)$ where $\left|w^{\prime}\right|=\left|w^{\prime \prime}\right|=m,\left|w_{0}\right|=n$.

$$
\begin{aligned}
a\left(\gamma_{0} w^{\prime}, w_{0} w^{\prime \prime}\right) & =\uparrow_{1 / n, 1+m / n+m}\left(t_{\gamma, w}\right)\left(\gamma_{0} w^{\prime}, w_{0} w^{\prime \prime}\right) \\
& =\left\langle w^{\prime}, w^{\prime \prime}\right\rangle^{-1}\left(t_{\gamma, w}\left(\gamma_{0}, w_{0}\right)\right)
\end{aligned}
$$

Then, we have $\gamma_{0}=\gamma, w_{0}=w$, and $\langle\epsilon, \epsilon\rangle \in\left\langle w^{\prime}, w^{\prime \prime}\right\rangle^{-1} t$, i.e., $\left\langle w^{\prime}, w^{\prime \prime}\right\rangle \in t$. Hence, $\left\langle p, \gamma w^{\prime}\right\rangle \Rightarrow\left\langle p^{\prime}, w w^{\prime \prime}\right\rangle$.

Case: $\left\langle p_{1}, m_{1}\right\rangle \stackrel{a_{1} \odot a_{2}}{\underset{\mathcal{P}^{\prime}}{\longrightarrow}}\left\langle p_{3}, m_{3}\right\rangle$ is obtained from $\left\langle p_{1}, m_{1}\right\rangle \underset{\mathcal{P}^{\prime}}{\stackrel{a_{1}}{\Rightarrow}}\left\langle p_{2}, m_{2}\right\rangle$ and $\left\langle p_{2}, m_{2}\right\rangle$

$\underset{\mathcal{P}^{\prime}}{\stackrel{a_{2}}{\Rightarrow}}\left\langle p_{3}, m_{3}\right\rangle$. Let $\langle\epsilon, \epsilon\rangle \in a_{1} \odot a_{2}\left(w_{1}, w_{3}\right)$. Then, $\langle\epsilon, \epsilon\rangle \in a_{1}\left(w_{1}, w_{2}\right) \circ a_{2}\left(w_{2}, w_{3}\right)$ for some

$w_{2}$. Since $a_{1}\left(w_{1}, w_{2}\right)$ and $a_{2}\left(w_{2}, w_{3}\right)$ are letter-to-letter transducers, $\langle\epsilon, \epsilon\rangle \in a_{1}\left(w_{1}, w_{2}\right)$ and $\langle\epsilon, \epsilon\rangle \in a_{2}\left(w_{2}, w_{3}\right)$. Then, we obtain $\left\langle p_{1}, w_{1}\right\rangle \underset{\mathcal{P}}{\Rightarrow}\left\langle p_{3}, w_{3}\right\rangle$ from the induction hypotheses. 